Pacific

Journal of

Mathematics

\title{
THE FIRST TERMS IN THE EXPANSION OF THE BERGMAN KERNEL IN HIGHER DEGREES
}

MARTIN PUCHOL AND JiALIN ZHU 


\title{
THE FIRST TERMS IN THE EXPANSION OF THE BERGMAN KERNEL IN HIGHER DEGREES
}

\author{
MARTin PuChOL AND JiALIN ZhU
}

\begin{abstract}
We establish the cancellation of the first $2 j$ terms in the diagonal asymptotic expansion of the restriction to the $(0,2 j)$-forms of the Bergman kernel associated to the $\operatorname{spin}^{c}$ Dirac operator on high tensor powers of a positive line bundle twisted by a (not necessarily holomorphic) complex vector bundle, over a compact Kähler manifold. Moreover, we give a local formula for the first and the second (nonzero) leading coefficients, as well as for the third assuming that the first two vanish.
\end{abstract}

\section{Introduction}

The Bergman kernel of a Kähler manifold endowed with a positive line bundle $L$ is the smooth kernel of the orthogonal projection on the kernel of the Kodaira Laplacian $\square^{L}=\bar{\partial}^{L} \bar{\partial}^{L, *}+\bar{\partial}^{L, *} \bar{\partial}^{L}$. The existence of a diagonal asymptotic expansion of the Bergman kernel associated with the $p$-th tensor power of $L$ when $p \rightarrow+\infty$ and the form of the leading term were proved in [Tian 1990; Zelditch 1998; Catlin 1999]. Moreover, the coefficients in this expansion encode geometric information about the underlying manifold, and therefore they have been studied closely: the second and third terms were computed by Lu [2000], X. Wang [2005], L. Wang [2003] and Ma and Marinescu [2012] in different degrees of generality (see also the recent paper [ $\mathrm{Xu} 2012])$. This asymptotic analysis plays an important role in various problems of Kähler geometry; see, for instance, [Donaldson 2001; Fine 2012]. We refer the reader to [Ma and Marinescu 2007] (henceforth abbreviated $[\mathrm{MM}])$ for a comprehensive study of the Bergman kernel and its applications. See also the survey [Ma 2011].

In fact, Dai, Liu and Ma [Dai et al. 2006] established the asymptotic development of the Bergman kernel in the symplectic case, using the heat kernel (see also [Ma and Marinescu 2006]). In [Charbonneau and Stern 2011], these asymptotics in the symplectic case have found an application in the study of the variation of Hodge structures of vector bundles. In that setting, the Bergman kernel is the kernel of a Kodaira-like Laplacian on a twisted bundle $L \otimes E$, where $E$ is a (not

MSC2010: 32A25, 53D50.

Keywords: Bergman kernel, quantization, asymptotic expansion. 
necessarily holomorphic) complex vector bundle. Because of that, the Bergman kernel is no longer supported in degree 0 (unlike it did in the Kähler case), and the asymptotic development of its restriction to the $(0,2 j)$-forms is related to the degree of 'nonholomorphicity' of $E$.

In this paper, we will show that the leading term in the asymptotics of the restriction to the $(0,2 j)$-forms of the Bergman kernel is of order $p^{\operatorname{dim} X-2 j}$ and we will compute it. That will lead to a local version of [Charbonneau and Stern 2011, Equation (1.3)], which is the main technical result of their paper; see Remark 0.6. After that, we will also compute the second term in the asymptotics, as well as the third term in the case where the first two vanish.

We now give more detail about our results. Let $(X, \omega, J)$ be a compact Kähler manifold of complex dimension $n$. Let $\left(L, h^{L}\right)$ be a holomorphic Hermitian line bundle on $X$, and $\left(E, h^{E}\right)$ a Hermitian complex vector bundle. We endow $\left(L, h^{L}\right)$ with its Chern (i.e., holomorphic and Hermitian) connection $\nabla^{L}$, and $\left(E, h^{E}\right)$ with a Hermitian connection $\nabla^{E}$, whose curvatures are $R^{L}=\left(\nabla^{L}\right)^{2}$ and $R^{E}=\left(\nabla^{E}\right)^{2}$.

Except in the beginning of Section $1 \mathrm{~A}$, we will always assume that $\left(L, h^{L}, \nabla^{L}\right)$ satisfies the prequantization condition

$$
\omega=\frac{\sqrt{-1}}{2 \pi} R^{L} .
$$

Let $g^{T X}(\cdot, \cdot)=\omega(\cdot, J \cdot)$ be the Riemannian metric on $T X$ induced by $\omega$ and $J$. It induces a metric $h^{\Lambda^{0, \bullet}}$ on $\Lambda^{0, \bullet}\left(T^{*} X\right):=\Lambda^{\bullet}\left(T^{*(0,1)} X\right)$; see Section 1A.

Let $L^{p}=L^{\otimes p}$ be the $p$-th tensor power of $L$. Let

$$
\Omega^{0, \bullet}\left(X, L^{p} \otimes E\right)=\mathscr{C}^{\infty}\left(X, \Lambda^{0, \bullet}\left(T^{*} X\right) \otimes L^{p} \otimes E\right)
$$

and $\bar{\partial}^{L^{p} \otimes E}: \Omega^{0, \bullet}\left(X, L^{p} \otimes E\right) \rightarrow \Omega^{0, \bullet+1}\left(X, L^{p} \otimes E\right)$ be the Dolbeault operator

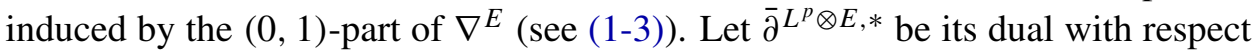
to the $L^{2}$-product. We set (see (1-6))

$$
D_{p}=\sqrt{2}\left(\bar{\partial}^{L^{p} \otimes E}+\bar{\partial}^{L^{p} \otimes E, *}\right),
$$

which exchanges odd and even forms.

Definition 0.1. Let

$$
P_{p}: \Omega^{0, \bullet}\left(X, L^{p} \otimes E\right) \rightarrow \operatorname{ker}\left(D_{p}\right)
$$

be the orthogonal projection onto the kernel of $D_{p}$. The operator $P_{p}$ is called the Bergman projection. It has a smooth kernel with respect to $d v_{X}(y)$, denoted by $P_{p}(x, y)$, which is called the Bergman kernel.

Remark 0.2. If $E$ is holomorphic, then by Hodge theory and the Kodaira vanishing theorem (see respectively [MM, Theorems 1.4.1 and 1.5.6]), we know that, for $p$ 
large enough, $P_{p}$ is the orthogonal projection $\mathscr{C}^{\infty}\left(X, L^{p} \otimes E\right) \rightarrow H^{0}\left(X, L^{p} \otimes E\right)$. Here, by [Ma and Marinescu 2002, Theorem 1.1], we just know that

$$
\operatorname{ker}\left(\left.D_{p}\right|_{\Omega^{0, \text { odd }}\left(X, L^{p} \otimes E\right)}\right)=0
$$

for $p$ large, so that $P_{p}: \Omega^{0, \text { even }}\left(X, L^{p} \otimes E\right) \rightarrow \operatorname{ker}\left(D_{p}\right)$. In particular, $P_{p}(x, x) \in$ $\mathscr{C}^{\infty}\left(X, \operatorname{End}\left(\Lambda^{0, \text { even }}\left(T^{*} X\right) \otimes E\right)\right)$.

By Theorem 1.3, $D_{p}$ is a Dirac operator, which enables us to apply this result: Theorem 0.3 [Dai et al. 2006, Theorem 1.1]. There exist

$$
\boldsymbol{b}_{r} \in \mathscr{C}^{\infty}\left(X, \operatorname{End}\left(\Lambda^{0, \operatorname{even}}\left(T^{*} X\right) \otimes E\right)\right)
$$

such that for any $k \in \mathbb{N}$ and $p \rightarrow+\infty$,

$$
p^{-n} P_{p}(x, x)=\sum_{r=0}^{k} \boldsymbol{b}_{r}(x) p^{-r}+O\left(p^{-k-1}\right),
$$

that is, for every $k, l \in \mathbb{N}$, there exists a constant $C_{k, l}>0$ such that for any $p \in \mathbb{N}$,

$$
\left|p^{-n} P_{p}(x, x)-\sum_{r=0}^{k} \boldsymbol{b}_{r}(x) p^{-r}\right|_{\mathscr{C}^{l}(X)} \leq C_{k, l} p^{-k-1} .
$$

Here $|\cdot|_{\mathscr{C}^{l}(X)}$ is the $\mathscr{C}^{l}$-norm for the variable $x \in X$.

To simplify the formulas, we denote by

$$
\mathscr{R}=\left(R^{E}\right)^{0,2} \in \Omega^{0,2}(X, \operatorname{End}(E))
$$

the $(0,2)$-part of $R^{E}$ (which is zero if $E$ is holomorphic). For $j \in \llbracket 1, n \rrbracket$, let

$$
I_{j}: \Lambda^{0, \bullet}\left(T^{*} X\right) \otimes E \rightarrow \Lambda^{0, j}\left(T^{*} X\right) \otimes E
$$

be the natural orthogonal projection. The first main result in this paper is:

Theorem 0.4. For any $k \in \mathbb{N}, k \geq 2 j$, we have when $p \rightarrow+\infty$,

$$
p^{-n} I_{2 j} P_{p}(x, x) I_{2 j}=\sum_{r=2 j}^{k} I_{2 j} \boldsymbol{b}_{r}(x) I_{2 j} p^{-r}+O\left(p^{-k-1}\right),
$$

and moreover,

$$
I_{2 j} \boldsymbol{b}_{2 j}(x) I_{2 j}=\frac{1}{(4 \pi)^{2 j}} \frac{1}{2^{2 j}(j !)^{2}} I_{2 j}\left(\mathscr{R}_{x}^{j}\right)\left(\mathscr{R}_{x}^{j}\right)^{*} I_{2 j},
$$

where $\left(\mathscr{R}_{x}^{j}\right)^{*}$ is the dual of $\mathscr{R}_{x}^{j}$ acting on $\left(\Lambda^{0, \bullet}\left(T^{*} X\right) \otimes E\right)_{x}$.

Theorem 0.4 leads immediately to: 
Corollary 0.5. Uniformly in $x \in X$, when $p \rightarrow+\infty$, we have

(0-13) $\operatorname{Tr}\left(\left(I_{2 j} P_{p} I_{2 j}\right)(x, x)\right)=\frac{1}{(4 \pi)^{2 j}} \frac{1}{2^{2 j}(j !)^{2}}\left\|\mathscr{R}_{x}^{j}\right\|^{2} p^{n-2 j}+O\left(p^{n-2 j-1}\right)$.

Remark 0.6. By integrating (0-13) over $X$, we get

$$
\operatorname{Tr}\left(I_{2 j} P_{p} I_{2 j}\right)=\frac{1}{(4 \pi)^{2 j}} \frac{1}{2^{2 j}(j !)^{2}}\left\|\Re^{j}\right\|_{L^{2}}^{2} p^{n-2 j}+O\left(p^{n-2 j-1}\right),
$$

which is the main technical result of [Charbonneau and Stern 2011, Equation (1.3)]; thus Corollary 0.5 can be viewed as a local version of it. The constant in (0-14) differs from the one in [ibid.] because our conventions are not the same as theirs (e.g., they chose $\omega=\sqrt{-1} R^{L}$ ).

Let $R_{\Lambda}^{E}:=-\sqrt{-1} \sum_{i} R^{E}\left(w_{i}, \bar{w}_{i}\right)$ for $\left(\bar{w}_{1}, \ldots, \bar{w}_{n}\right)$ an orthonormal frame of $T^{(0,1)} X$. Let $R^{T X}$ be the curvature of the Levi-Civita connection $\nabla^{T X}$ of $\left(X, g^{T X}\right)$, and for $\left(e_{1}, \ldots, e_{2 n}\right)$ an orthonormal frame of $T X$, let $r^{X}=-\sum_{i, j}\left\langle R^{T X}\left(e_{i}, e_{j}\right) e_{i}, e_{j}\right\rangle$
be the scalar curvature of $X$.

For $j, k \in \mathbb{N}$ and $j \geq k$, we also define $\mathrm{C}_{j}(k)$ by

$$
\mathrm{C}_{j}(k):=\frac{1}{(4 \pi)^{j}} \frac{1}{2^{k} k !} \frac{1}{\prod_{s=k+1}^{j}(2 s+1)}
$$

with the convention that $\prod_{s \in \varnothing}=1$.

Let $\nabla^{\Lambda^{0, \bullet}}$ be the connection on $\Lambda^{0, \bullet}\left(T^{*} X\right)$ induced by $\nabla^{T X}$. Let $\nabla^{\Lambda^{0, \bullet} \otimes E}$ be the connection on $\Lambda^{0, \bullet}\left(T^{*} X\right) \otimes E$ induced by $\nabla^{E}$ and $\nabla^{\Lambda^{0, \bullet}}$, and let $\Delta^{\Lambda^{0, \bullet} \otimes E}$ be the associated Laplacian. For precise definitions, see Section 1A.

For every operator $A$ acting on a Hermitian space, we define the positive (not necessarily definite) operator and the symmetric operator associated to $A$ as

$$
\operatorname{Pos}[A]=A A^{*} \quad \text { and } \quad \operatorname{Sym}[A]=A+A^{*} .
$$

Finally, to simplify the notation, we define $\mathcal{T}_{0}(j), \mathcal{T}_{1}(j), \mathcal{T}_{2}(j)$ and $\mathcal{T}_{3}(j)$ as:

- $\mathcal{T}_{0}(0)=0$, and for $j \geq 1$,

$(0-17) \quad \mathcal{T}_{0}(j)=\frac{1}{\sqrt{2 \pi}} \sum_{i=0}^{n} \sum_{k=0}^{j-1} I_{2 j}\left(\mathrm{C}_{j}(j)-\mathrm{C}_{j}(k)\right) \mathscr{R}_{x}^{j-k-1}\left(\nabla_{\bar{w}_{i}}^{\Lambda^{0} \bullet \otimes E} \mathscr{R}.\right)(x) \mathscr{R}_{x}^{k} I_{0}$. 
- $\mathcal{T}_{1}(0)=\mathcal{T}_{1}(1)=0$, and for $j \geq 2$,

$(0-18) \quad \mathcal{T}_{1}(j)=\frac{I_{2 j}}{2 \pi} \sum_{q=0}^{j-2} \sum_{m=0}^{q}\left\{\left(\mathrm{C}_{j}(j)-\mathrm{C}_{j}(q+1)\right)\right.$

$$
\begin{array}{r}
\times \mathscr{R}_{x}^{j-(q+2)}\left(\nabla_{\bar{w}_{i}}^{\Lambda^{0, \bullet} \otimes E} \mathscr{R} .\right)(x) \mathscr{R}_{x}^{q-m}\left(\nabla_{w_{i}}^{\Lambda^{0, \bullet} \otimes E} \mathscr{R} .\right)(x) \mathscr{R}_{x}^{m} \\
+\mathrm{C}_{j}(m)\left[\prod_{s=q+2}^{j}\left(1+\frac{1}{2 s}\right)-1\right] \\
\left.\times \mathscr{R}_{x}^{j-(q+2)}\left(\nabla_{w_{i}}^{\Lambda^{0, \bullet} \otimes E_{\mathscr{R}}}\right)(x) \mathscr{R}_{x}^{q-m}\left(\nabla_{\bar{w}_{i}}^{\Lambda^{0, \bullet} \otimes E_{\mathscr{R}}}\right)(x) \mathscr{R}_{x}^{m}\right\} I_{0},
\end{array}
$$

- $\mathcal{T}_{2}(0)=0$, and for $j \geq 1$,

$$
\mathcal{T}_{2}(j)=\frac{1}{4 \pi} I_{2 j} \sum_{k=0}^{j-1}\left\{\left(\mathrm{C}_{j}(k)-\mathrm{C}_{j}(j)\right) \mathscr{R}_{x}^{j-(k+1)}\left(\Delta^{\Lambda^{0, \bullet} \otimes E} \mathscr{R} .\right)(x) \mathscr{R}_{x}^{k}\right\} I_{0},
$$

- for $j \geq 0$,

$(0-20) \quad \mathcal{T}_{3}(j)=I_{2 j} \sum_{k=0}^{j} \mathscr{R}_{x}^{j-k}\left[\frac{1}{6}\left(\mathrm{C}_{j+1}(j+1)-\frac{\mathrm{C}_{j}(k)}{2 \pi(2 k+1)}\right) r_{x}^{X}\right.$

$$
\left.-\frac{\mathrm{C}_{j}(k)}{4 \pi(2 k+1)} \sqrt{-1} R_{\Lambda, x}^{E}\right] \mathscr{R}_{x}^{k} I_{0} .
$$

Our second goal is to compute the second term in the expansion (0-11).

Theorem 0.7. We can decompose $I_{2 j} \boldsymbol{b}_{2 j+1}(x) I_{2 j}$ as the sum of four terms:

$(0-21) \quad I_{2 j} \boldsymbol{b}_{2 j+1}(x) I_{2 j}$

$$
=\operatorname{Pos}\left[\mathcal{T}_{0}(j)\right]+\mathrm{C}_{j}(j) \operatorname{Sym}\left[\left(\mathcal{T}_{1}(j)+\mathcal{T}_{2}(j)+\mathcal{T}_{3}(j)\right)\left(\mathscr{R}_{x}^{j}\right)^{*} I_{2 j}\right] .
$$

For instance, for $j=1$, using the fact that $\left(R_{\Lambda}^{E}\right)^{*}=R_{\Lambda}^{E}$, we find

$(0-22) \quad 128 \pi^{3} I_{2} \boldsymbol{b}_{3}(x) I_{2}$

$$
\begin{aligned}
= & \frac{1}{9} \operatorname{Pos}\left[I_{2} \sum_{i=0}^{n}\left(\nabla_{\bar{w}_{i}}^{\Lambda^{0} \bullet \otimes E} \mathscr{R} .\right)(x) I_{0}\right]-\frac{1}{6} \operatorname{Sym}\left[I_{2}\left(\Delta^{\Lambda^{0, \bullet} \otimes E} \mathscr{R} .\right)(x) \mathscr{R}_{x}^{*} I_{2}\right] \\
& -\frac{\sqrt{-1}}{6} I_{2}\left(R_{\Lambda}^{E} \mathscr{R}_{x} \mathscr{R}_{x}^{*}+\mathscr{R}_{x} \mathscr{R}_{x}^{*} R_{\Lambda}^{E}\right) I_{2}-\frac{2 \sqrt{-1}}{3} I_{2} \mathscr{R}_{x} R_{\Lambda}^{E} \mathscr{R}_{x}^{*} I_{2}-\frac{r_{x}^{X}}{4} I_{2} \mathscr{R}_{x} \mathscr{R}_{x}^{*} I_{2} .
\end{aligned}
$$

The last goal of this paper is to compute the third term in the expansion (0-11), assuming that the first two vanish.

Theorem 0.8. Let $j \in \llbracket 1, n \rrbracket$. If

$$
I_{2 j} \boldsymbol{b}_{2 j}(x) I_{2 j}=I_{2 j} \boldsymbol{b}_{2 j+1}(x) I_{2 j}=0,
$$


then $\mathcal{T}_{3}$ equals

$$
\mathcal{T}_{3}^{\prime}(j):=-\sqrt{-1} I_{2 j} \sum_{k=0}^{j} \frac{\mathrm{C}_{j}(k)}{4 \pi(2 k+1)} \mathscr{R}_{x}^{j-k} R_{\Lambda, x}^{E} \Re_{x}^{k} I_{0},
$$

and

$$
I_{2 j} \boldsymbol{b}_{2 j+2}(x) I_{2 j}=\operatorname{Pos}\left[\mathcal{T}_{1}(j)+\mathcal{T}_{2}(j)+\mathcal{T}_{3}^{\prime}(j)\right] .
$$

Theorems $0.4,0.7$ and 0.8 yield to:

Corollary 0.9. We have

$$
I_{2 j} P_{p}(x, x) I_{2 j}=O\left(p^{n-2 j-3}\right) \Longleftrightarrow\left\{\begin{array}{l}
\mathscr{R}_{x}^{j}=0, \\
\mathcal{T}_{0}(j)=0, \\
\mathcal{T}_{1}(j)+\mathcal{T}_{2}(j)+\mathcal{T}_{3}^{\prime}(j)=0 .
\end{array}\right.
$$

This paper is organized as follows. In Section 1 we compute the square of $D_{p}$ and use a local trivialization to rescale it, and then give the Taylor expansion of the rescaled operator. In Section 2, we use this expansion to give a formula for the coefficients $\boldsymbol{b}_{r}$ appearing in (0-7), which will lead to a proof of Theorem 0.4. In Section 3, we prove Theorem 0.7 using the formula for $\boldsymbol{b}_{r}$. Finally, in Section 4, we prove Theorem 0.8 using the techniques and results of the preceding sections.

In this whole paper, when an index variable appears twice in a single term, it means that we are summing over all its possible values.

\section{Rescaling $D_{p}^{2}$ and Taylor expansion}

In this section, we follow the method of [MM, Chapter 4] that enables to prove the existence of $\boldsymbol{b}_{r}$ in (0-7) in the case of a holomorphic vector bundle $E$, and that still applies here (as pointed out in [MM, Section 8.1.1]). Then, in Sections 2 and 3, we will use this approach to understand $I_{2 j} \boldsymbol{b}_{r} I_{2 j}$ and prove Theorems 0.4 and 0.7.

In Section 1A, we will first prove Theorem 1.3, and then give a formula for the square of $D_{p}$, which will be the starting point of our approach.

In Section $1 \mathrm{~B}$, we will rescale the operator $D_{p}^{2}$ to get an operator $\mathscr{L}_{t}$, and then give the Taylor expansion of the rescaled operator.

In Section 1C, we will study more precisely the limit operator $\mathscr{L}_{0}$.

1A. The square of $D_{p}$. For further details on the material of this subsection, the lector can read [MM]. First of all let us give some notation.

The Riemannian volume form of $\left(X, g^{T X}\right)$ is given by $d v_{X}=\omega^{n} / n !$. We will denote by $\langle\cdot, \cdot\rangle$ the $\mathbb{C}$-bilinear form on $T X \otimes \mathbb{C}$ induced by $g^{T X}$.

For the rest of Section 1A, we will fix $\left(w_{1}, \ldots, w_{n}\right)$ a local orthonormal frame of $T^{(1,0)} X$ with dual frame $\left(w^{1}, \ldots, w^{n}\right)$. Then $\left(\bar{w}_{1}, \ldots, \bar{w}_{n}\right)$ is a local orthonormal 
frame of $T^{(0,1)} X$ whose dual frame is denoted by $\left(\bar{w}^{1}, \ldots, \bar{w}^{n}\right)$, and the vectors

$$
e_{2 j-1}=\frac{1}{\sqrt{2}}\left(w_{j}+\bar{w}_{j}\right) \quad \text { and } \quad e_{2 j}=\frac{\sqrt{-1}}{\sqrt{2}}\left(w_{j}-\bar{w}_{j}\right)
$$

form a local orthonormal frame of $T X$.

We choose the Hermitian metric $h^{\Lambda^{0, \bullet}}$ on $\Lambda^{0, \bullet}\left(T^{*} X\right):=\Lambda^{\bullet}\left(T^{*(0,1)} X\right)$ such that $\left\{\bar{w}^{j_{1}} \wedge \cdots \wedge \bar{w}^{j_{k}}: 1 \leq j_{1}<\cdots<j_{k} \leq n\right\}$ is an orthonormal frame of $\Lambda^{0, \bullet}\left(T^{*} X\right)$.

For any Hermitian bundle $\left(F, h^{F}\right)$ over $X$, let $\mathscr{C}^{\infty}(X, F)$ be the space of smooth sections of $F$. It is endowed with the $L^{2}$-Hermitian metric

$$
\left\langle s_{1}, s_{2}\right\rangle=\int_{X}\left\langle s_{1}(x), s_{2}(x)\right\rangle_{h^{F}} d v_{x}(x) .
$$

The corresponding norm will be denoted by $\|\cdot\|_{L^{2}}$, and the completion of $\mathscr{C}^{\infty}(X, F)$ with respect to this norm by $L^{2}(X, F)$.

Let $\bar{\partial}^{E}$ be the Dolbeault operator of $E$. It is the $(0,1)$-part of the connection $\nabla^{E}$

$$
\bar{\partial}^{E}:=\left(\nabla^{E}\right)^{0,1}: \mathscr{C}^{\infty}(X, E) \rightarrow \mathscr{C}^{\infty}\left(X, T^{*(0,1)} X \otimes E\right) .
$$

We extend it to get an operator

$$
\bar{\partial}^{E}: \Omega^{0, \bullet}(X, E) \rightarrow \Omega^{0, \bullet+1}(X, E)
$$

by the Leibniz formula: for $s \in \mathscr{C}^{\infty}(X, E)$ and $\alpha \in \mathscr{C}^{\infty}\left(X, \Lambda^{0, \bullet}\left(T^{*} X\right)\right)$ homogeneous,

$$
\bar{\partial}^{E}(\alpha \otimes s)=(\bar{\partial} \alpha) \otimes s+(-1)^{\operatorname{deg} \alpha} \alpha \otimes \bar{\partial}^{E} s .
$$

We can now define the operator

$$
D^{E}=\sqrt{2}\left(\bar{\partial}^{E}+\bar{\partial}^{E, *}\right): \Omega^{0, \bullet}(X, E) \rightarrow \Omega^{0, \bullet}(X, E),
$$

where the dual is taken with respect to the $L^{2}$-norm associated with the Hermitian metrics $h^{\Lambda^{0, \bullet}}$ and $h^{E}$.

Let $\nabla^{\Lambda\left(T^{*} X\right)}$ be the connection on $\Lambda\left(T^{*} X\right)$ induced by the Levi-Civita connection $\nabla^{T X}$ of $X$. Since $X$ is Kähler, $\nabla^{T X}$ preserves $T^{(0,1)} X$ and $T^{(1,0)} X$. Thus, it induces a connection $\nabla^{T^{*(0,1)} X}$ on $T^{*(0,1)} X$, and then a Hermitian connection $\nabla^{\Lambda^{0, \bullet}}$ on $\Lambda^{0, \bullet}\left(T^{*} X\right)$. We then have that for any $\alpha \in \mathscr{C}^{\infty}\left(X, \Lambda^{0, \bullet}\left(T^{*} X\right)\right)$,

$$
\nabla^{\Lambda^{0, \bullet}} \alpha=\nabla^{\Lambda\left(T^{*} X\right)} \alpha
$$

Note the important fact that $\nabla^{\Lambda\left(T^{*} X\right)}$ preserves the bi-grading on $\Lambda^{\bullet \bullet \bullet}\left(T^{*} X\right)$.

Let $\nabla^{\Lambda^{0 \bullet \bullet} \otimes E}:=\nabla^{\Lambda^{0, \bullet}} \otimes 1+1 \otimes \nabla^{E}$ be the connection on $\Lambda^{0, \bullet}\left(T^{*} X\right) \otimes E$ induced by $\nabla^{\Lambda^{0, \bullet}}$ and $\nabla^{E}$. 
Proposition 1.1. On $\Omega^{0, \bullet}(X, E)$, we have

$$
\begin{aligned}
\bar{\partial}^{E} & =\bar{w}^{j} \wedge \nabla_{\bar{w}_{j}}^{\Lambda^{0, \bullet} \otimes E}, \\
\bar{\partial}^{E, *} & =-i_{\bar{w}_{j}} \nabla_{w_{j}}^{\Lambda^{0, \bullet} \otimes E} .
\end{aligned}
$$

Proof. We still denote by $\nabla^{E}$ the extension of the connection $\nabla^{E}$ to $\Omega^{\bullet \bullet \bullet}(X, E)$ by the usual formula $\nabla^{E}(\alpha \otimes s)=d \alpha \otimes s+(-1)^{\operatorname{deg} \alpha} \alpha \wedge \nabla^{E} s$ for $s \in \mathscr{C}^{\infty}(X, E)$ and $\alpha \in \mathscr{C}^{\infty}\left(X, \Lambda\left(T^{*} X\right)\right)$ homogeneous. We know that $d=\varepsilon \circ \nabla^{\Lambda\left(T^{*} X\right)}$ where $\varepsilon$ is the exterior multiplication (see [MM, Equation (1.2.44)]), so we get that $\nabla^{E}=\varepsilon \circ \nabla^{\Lambda\left(T^{*} X\right) \otimes E}$. Using (1-7), it follows that

$$
\bar{\partial}^{E}=\left(\nabla^{E}\right)^{0,1}=\bar{w}^{j} \wedge \nabla_{\bar{w}_{j}}^{\Lambda^{0, \bullet} \otimes E},
$$

which is the first part of (1-8).

The second part of our proposition follows classically from the first by exactly the same computation as in [MM, Lemma 1.4.4].

Definition 1.2. Let $v=v^{1,0}+v^{0,1} \in T X=T^{(1,0)} X \oplus T^{(0,1)} X$, and $\bar{v}^{(0,1), *} \in T^{*(0,1)} X$ the dual of $v^{1,0}$ for $\langle\cdot, \cdot\rangle$. We define the Clifford action of $T X$ on $\Lambda^{0, \bullet}\left(T^{*} X\right)$ by

$$
c(v)=\sqrt{2}\left(\bar{v}^{(0,1), *} \wedge-i_{v^{0,1}}\right) .
$$

We verify easily that for $u, v \in T X$,

$$
c(u) c(v)+c(v) c(u)=-2\langle u, v\rangle,
$$

and that for any skew-adjoint endomorphism $A$ of $T X$,

$$
\begin{aligned}
\frac{1}{4}\left\langle A e_{i}, e_{j}\right\rangle c\left(e_{i}\right) c\left(e_{j}\right)= & -\frac{1}{2}\left\langle A w_{j}, \bar{w}_{j}\right\rangle+\left\langle A w_{\ell}, \bar{w}_{m}\right\rangle \bar{w}^{m} \wedge i_{\bar{w}_{\ell}} \\
& +\frac{1}{2}\left\langle A w_{\ell}, w_{m}\right\rangle i_{\bar{w}_{\ell}} i_{\bar{w}_{m}}+\frac{1}{2}\left\langle A \bar{w}_{\ell}, \bar{w}_{m}\right\rangle \bar{w}^{\ell} \wedge \bar{w}^{m} \wedge .
\end{aligned}
$$

Let $\nabla^{\text {det }}$ be the Chern connection of $\operatorname{det}\left(T^{(1,0)} X\right):=\Lambda^{n}\left(T^{(1,0)} X\right)$, and $\nabla^{\mathrm{Cl}}$ the Clifford connection on $\Lambda^{0, \bullet}\left(T^{*} X\right)$ induced by $\nabla^{T X}$ and $\nabla^{\operatorname{det}}$ (see [MM, Equation (1.3.5)]). We also denote by $\nabla^{\mathrm{Cl}}$ the connection on $\Lambda^{0, \bullet}\left(T^{*} X\right) \otimes E$ induced by $\nabla^{\mathrm{Cl}}$ and $\nabla^{E}$. By [loc. cit.], (1-11) and the fact that $\nabla^{\text {det }}$ is holomorphic, we get

$$
\nabla^{\mathrm{Cl}}=\nabla^{\Lambda^{0, \bullet}} \text {. }
$$

Let $D^{c, E}$ be the associated $\operatorname{spin}^{c}$ Dirac operator

$$
D^{c, E}=\sum_{j=1}^{2 n} c\left(e_{j}\right) \nabla_{e_{j}}^{\mathrm{Cl}}: \Omega^{0, \bullet}(X, E) \rightarrow \Omega^{0, \bullet}(X, E) .
$$

By (1-8) and (1-12), we have:

Theorem 1.3. $D^{E}$ is equal to the $\operatorname{spin}^{c}$ Dirac operator $D^{c, E}$ acting on $\Omega^{0, \bullet}(X, E)$. 
Remark 1.4. Note that all the results proved in the beginning of this subsection hold without assuming the prequantization condition (0-1), but from now on we will use it.

Let $\left(F, h^{F}\right)$ be a Hermitian vector bundle on $X$ and let $\nabla^{F}$ be a Hermitian connection on $F$. Then the Bochner Laplacian $\Delta^{F}$ acting on $\mathscr{C}^{\infty}(X, F)$ is defined by

$$
\Delta^{F}=-\sum_{j=1}^{2 n}\left(\left(\nabla_{e_{j}}^{F}\right)^{2}-\nabla_{\nabla_{e_{j}}^{T X}}^{F}\right) .
$$

On $\Omega^{0, \bullet}(X)$, we define the number operator $\mathcal{N}$ by

$$
\left.\mathcal{N}\right|_{\Omega^{0, j}(X)}=j,
$$

and we also denote by $\mathcal{N}$ the operator $\mathcal{N} \otimes 1$ acting on $\Omega^{0, \bullet}(X, F)$.

The bundle $L^{p}$ is endowed with the connection $\nabla^{L^{p}}$ induced by $\nabla^{L}$ (which is also its Chern connection). Let $\nabla^{L^{p} \otimes E}:=\nabla^{L^{p}} \otimes 1+1 \otimes \nabla^{E}$ be the connection on $L^{p} \otimes E$ induced by $\nabla^{L}$ and $\nabla^{E}$. We will denote

$$
D_{p}=D^{L^{p} \otimes E} .
$$

Theorem 1.5. The square of $D_{p}$ is given by

$$
\begin{aligned}
& D_{p}^{2}=\Delta^{\Lambda^{0, \bullet} \otimes L^{p} \otimes E}-R^{E}\left(w_{j}, \bar{w}_{j}\right)-2 \pi p n+4 \pi p \mathcal{N} \\
&+2\left(R^{E}+\frac{1}{2} R^{\mathrm{det}}\right)\left(w_{\ell}, \bar{w}_{m}\right) \bar{w}^{m} \wedge i_{\bar{w}_{\ell}}+R^{E}\left(w_{\ell}, w_{m}\right) i_{\bar{w}_{\ell}} i_{\bar{w}_{m}} \\
&+R^{E}\left(\bar{w}_{\ell}, \bar{w}_{m}\right) \bar{w}^{\ell} \wedge \bar{w}^{m}
\end{aligned}
$$

Proof. By Theorem 1.3, we can use [MM, Theorem 1.3.5]

$$
D_{p}^{2}=\Delta^{\mathrm{Cl}}+\frac{1}{4} r^{X}+\frac{1}{2}\left(R^{L^{p} \otimes E}+\frac{1}{2} R^{\mathrm{det}}\right)\left(e_{i}, e_{j}\right) c\left(e_{i}\right) c\left(e_{j}\right),
$$

where $r^{X}$ is the scalar curvature of $X$. From (1-12), we see that $\Delta^{\mathrm{Cl}}=\Delta^{\Lambda^{0, \bullet} \otimes L^{p} \otimes E}$. Moreover, $r^{X}=2 R^{\operatorname{det}}\left(w_{j}, \bar{w}_{j}\right)$ and $R^{L^{p} \otimes E}=R^{E}+p R^{L}$. Using the equivalent of (1-11) for 2-forms (substituting $A(\cdot, \cdot)$ for $\langle A \cdot, \cdot\rangle$ ) and the fact that $R^{L}$ and $R^{\text {det }}$ are (1,1)-forms, (1-18) reads

$$
\begin{array}{r}
D_{p}^{2}=\Delta^{\Lambda^{0, \bullet} \otimes L^{p} \otimes E}+\frac{1}{2} R^{\operatorname{det}}\left(w_{j}, \bar{w}_{j}\right)-\left(R^{E}\left(w_{j}, \bar{w}_{j}\right)+p R^{L}\left(w_{j}, \bar{w}_{j}\right)+\frac{1}{2} R^{\operatorname{det}}\left(w_{j}, \bar{w}_{j}\right)\right) \\
+2\left(R^{E}+p R^{L}+\frac{1}{2} R^{\operatorname{det}}\right)\left(w_{\ell}, \bar{w}_{m}\right) \bar{w}^{m} \wedge i_{\bar{w}_{\ell}}+R^{E}\left(w_{\ell}, w_{m}\right) i_{\bar{w}_{\ell}} i_{\bar{w}_{m}} \\
+R^{E}\left(\bar{w}_{\ell}, \bar{w}_{m}\right) \bar{w}^{\ell} \wedge \bar{w}^{m} .
\end{array}
$$

Thanks to (0-1), we have $R^{L}\left(w_{\ell}, \bar{w}_{m}\right)=2 \pi \delta_{\ell m}$. Moreover, $\mathcal{N}=\sum_{\ell} \bar{w}^{\ell} \wedge i_{\bar{w}_{\ell}}$, thus we get Theorem 1.5. 
1B. Rescaling $D_{p}^{\mathbf{2}}$. In this subsection, we rescale $D_{p}^{2}$, but to do this we must define it on a vector space. Therefore, we will use normal coordinates to transfer the problem on the tangent space to $X$ at a fixed point. Then we give a Taylor expansion of the rescaled operator, but the problem is that each operator acts on a different space, namely,

$$
\mathrm{E}_{p}:=\Lambda^{0, \bullet}\left(T^{*} X\right) \otimes L^{p} \otimes E,
$$

so we must first handle this issue.

Fix $x_{0} \in X$. For the rest of this paper, we fix $\left\{w_{j}\right\}$ an orthonormal basis of $T_{x_{0}}^{(1,0)} X$, with dual basis $\left\{w^{j}\right\}$, and we construct an orthonormal basis $\left\{e_{i}\right\}$ of $T_{x_{0}} X$ from $\left\{w_{j}\right\}$ as in (1-1).

For $\varepsilon>0$, we denote by $B^{X}\left(x_{0}, \varepsilon\right)$ and $B^{T_{x_{0}} X}(0, \varepsilon)$ the open balls in $X$ and $T_{x_{0}} X$ with center $x_{0}$ and 0 and radius $\varepsilon$. If $\exp _{x_{0}}^{X}$ is the Riemannian exponential of $X$, then for $\varepsilon$ small enough, $Z \in B^{T_{x_{0}} X}(0, \varepsilon) \mapsto \exp _{x_{0}}^{X}(Z) \in B^{X}\left(x_{0}, \varepsilon\right)$ is a diffeomorphism, which gives local coordinates by identifying $T_{x_{0}} X$ with $\mathbb{R}^{2 n}$ via the orthonormal basis $\left\{e_{i}\right\}$ :

$$
\left(Z_{1}, \ldots, Z_{2 n}\right) \in \mathbb{R}^{2 n} \mapsto \sum_{i} Z_{i} e_{i} \in T_{x_{0}} X .
$$

From now on, we will always identify $B^{T_{x_{0}} X}(0, \varepsilon)$ and $B^{X}\left(x_{0}, \varepsilon\right)$. Note that in this identification, the radial vector field $\mathcal{R}=\sum_{i} Z_{i} e_{i}$ becomes $\mathcal{R}=Z$, so $Z$ can be
viewed as a point or as a tangent vector.

For $Z \in B^{T_{x_{0}} X}(0, \varepsilon)$, we identify $\left(L_{Z}, h_{Z}^{L}\right),\left(E_{Z}, h_{Z}^{E}\right)$ and $\left(\Lambda_{Z}^{0, \bullet}\left(T^{*} X\right), h_{Z}^{\Lambda^{0, \bullet}}\right)$ with $\left(L_{x_{0}}, h_{x_{0}}^{L}\right),\left(E_{x_{0}}, h_{x_{0}}^{E}\right)$ and $\left(\Lambda^{0, \bullet}\left(T_{x_{0}}^{*} X\right), h_{x_{0}}^{\Lambda^{0, \bullet}}\right)$ by parallel transport with respect to the connection $\nabla^{L}, \nabla^{E}$ and $\nabla^{\Lambda^{0, \bullet}}$ along the geodesic ray $t \in[0,1] \mapsto t Z$. We denote by $\Gamma^{L}, \Gamma^{E}$ and $\Gamma^{\Lambda^{0, \bullet}}$ the corresponding connection forms of $\nabla^{L}, \nabla^{E}$ and $\nabla^{\Lambda^{0, \bullet}}$. Remark 1.6. As $\nabla^{\Lambda^{0, \bullet}}$ preserves the degree, the identification between $\Lambda^{0, \bullet}\left(T^{*} X\right)$ and $\Lambda^{0, \bullet}\left(T_{x_{0}}^{*} X\right)$ is compatible with the degree. Thus, $\Gamma_{Z}^{\Lambda^{0, \bullet}} \in \bigoplus_{j} \operatorname{End}\left(\Lambda^{0, j}\left(T^{*} X\right)\right)$.

Let $S_{L}$ be a unit vector of $L_{x_{0}}$. It gives an isometry $L_{x_{0}}^{p} \simeq \mathbb{C}$, which induces an isometry

$$
\mathrm{E}_{p, x_{0}} \simeq\left(\Lambda^{0, \bullet}\left(T^{*} X\right) \otimes E\right)_{x_{0}}=: \mathbb{E}_{x_{0}} .
$$

Thus, in our trivialization, $D_{p}^{2}$ acts on $\mathbb{E}_{x_{0}}$, but this action may a priori depend on the choice of $S_{L}$. In fact, since the operator $D_{p}^{2}$ takes values in $\operatorname{End}\left(\mathrm{E}_{p, x_{0}}\right)$ which is canonically isomorphic to $\operatorname{End}(\mathbb{E})_{x_{0}}$ (by the natural identification $\left.\operatorname{End}\left(L^{p}\right) \simeq \mathbb{C}\right)$, all our formulas do not depend on this choice.

Let $d v_{T X}$ be the Riemannian volume form of $\left(T_{x_{0}} X, g^{T_{x_{0}}} X\right)$, and let $\kappa(Z)$ be the smooth positive function defined for $|Z| \leq \varepsilon$ by

$$
d v_{X}(Z)=\kappa(Z) d v_{T X}(Z),
$$


with $\kappa(0)=1$.

Definition 1.7. We denote by $\nabla_{U}$ the ordinary differentiation operator in the direction $U$ on $T_{x_{0}} X$. For $s \in \mathscr{C}^{\infty}\left(\mathbb{R}^{2 n}, \mathbb{E}_{x_{0}}\right)$ and $t=1 / \sqrt{p}$, set

$$
\begin{aligned}
\left(S_{t} s\right)(Z) & =s(Z / t) \\
\nabla_{t} & =t S_{t}^{-1} \kappa^{1 / 2} \nabla^{\mathrm{Cl}_{0}} \kappa^{-1 / 2} S_{t}, \\
\nabla_{0} & =\nabla+\frac{1}{2} R_{x_{0}}^{L}(Z, \cdot), \\
\mathscr{L}_{t} & =t^{2} S_{t}^{-1} \kappa^{1 / 2} D_{p}^{2} \kappa^{-1 / 2} S_{t}, \\
\mathscr{L}_{0} & =-\sum_{i}\left(\nabla_{0, e_{i}}\right)^{2}+4 \pi \mathcal{N}-2 \pi n .
\end{aligned}
$$

Let $\|\cdot\|_{L^{2}}$ be the $L^{2}$-norm induced by $h^{\mathrm{E}_{x_{0}}}$ and $d v_{T X}$. We can now state the key result in our approach to Theorems 0.4 and 0.7 :

Theorem 1.8. There exist second-order formally self-adjoint (with respect to $\|\cdot\|_{L^{2}}$ ) differential operators $\mathcal{O}_{r}$ with polynomial coefficients such that for all $m \in \mathbb{N}$,

$$
\mathscr{L}_{t}=\mathscr{L}_{0}+\sum_{r=1}^{m} t^{r} \mathcal{O}_{r}+O\left(t^{m+1}\right) .
$$

Furthermore, each $\mathcal{O}_{r}$ can be decomposed as

$$
\mathcal{O}_{r}=\mathcal{O}_{r}^{0}+\mathcal{O}_{r}^{+2}+\mathcal{O}_{r}^{-2}
$$

where $\mathcal{O}_{r}^{k}$ changes the degree of the form it acts on by $k$.

Proof. The first part of the theorem (i.e., (1-23)) is contained in [Ma and Marinescu 2008, Theorem 1.4]. We will briefly recall how they obtained this result.

Let $\Phi_{E}$ be the smooth self-adjoint section of $\operatorname{End}\left(\mathbb{E}_{x_{0}}\right)$ on $B^{T_{x_{0}} X}(0, \varepsilon)$ :

$$
\begin{aligned}
\Phi_{E}=-R^{E}\left(w_{j}, \bar{w}_{j}\right)+2\left(R^{E}+\frac{1}{2} R^{\operatorname{det}}\right)\left(w_{\ell}, \bar{w}_{m}\right) \bar{w}^{m} \wedge i_{\bar{w}_{\ell}} \\
+R^{E}\left(w_{\ell}, w_{m}\right) i_{\bar{w}_{\ell}} i_{\bar{w}_{m}}+R^{E}\left(\bar{w}_{\ell}, \bar{w}_{m}\right) \bar{w}^{\ell} \wedge \bar{w}^{m} .
\end{aligned}
$$

We can see that we can decompose $\Phi_{E}$ as $\Phi_{E}^{0}+\Phi_{E}^{+2}+\Phi_{E}^{-2}$, where

$$
\Phi_{E}^{0}=R^{E}\left(w_{j}, \bar{w}_{j}\right)+2\left(R^{E}+\frac{1}{2} R^{\mathrm{det}}\right)\left(w_{\ell}, \bar{w}_{m}\right) \bar{w}^{m} \wedge i_{\bar{w}_{\ell}} \text { preserves the degree, }
$$

$\Phi_{E}^{+2}=R^{E}\left(\bar{w}_{\ell}, \bar{w}_{m}\right) \bar{w}^{\ell} \wedge \bar{w}^{m}$ rises the degree by 2 ,

$\Phi_{E}^{-2}=R^{E}\left(w_{\ell}, w_{m}\right) i_{\bar{w}_{\ell}} i_{\bar{w}_{m}}$ lowers the degree by 2 .

Using Theorem 1.5, we find that

$$
D_{p}^{2}=\Delta^{\Lambda^{0, \bullet} \otimes L^{p} \otimes E}+p(-2 \pi n+4 \pi \mathcal{N})+\Phi_{E} .
$$


Let $g_{i j}(Z)=g^{T X}\left(e_{i}, e_{j}\right)(Z)$ and $\left(g^{i j}(Z)\right)_{i j}$ be the inverse of the matrix $\left(g_{i j}(Z)\right)_{i j}$. Let $\left(\nabla_{e_{i}}^{T X} e_{j}\right)(Z)=\Gamma_{i j}^{k}(Z) e_{k}$. As in [MM, Equation (4.1.34)], by (1-22) and (1-27), we get

$$
\begin{aligned}
\nabla_{t, \cdot} & =\kappa^{1 / 2}(t Z)\left(\nabla .+t \Gamma_{t Z}^{\Lambda^{0, \bullet}}+\frac{1}{t} \Gamma_{t Z}^{L}+t \Gamma_{t Z}^{E}\right) \kappa^{-1 / 2}(t Z), \\
\mathscr{L}_{t} & =-g^{i j}(t Z)\left(\nabla_{t, e_{i}} \nabla_{t, e_{j}}-t \Gamma_{i j}^{k}(t Z) \nabla_{t, e_{k}}\right)-2 \pi n+4 \pi \mathcal{N}+t^{2} \Phi_{E}(t Z) .
\end{aligned}
$$

Moreover, $\kappa=\left(\operatorname{det}\left(g_{i j}\right)\right)^{1 / 2}$, thus we can prove equation (1-23) as in [MM, Theorem 4.1.7] by taking the Taylor expansion of each term appearing in (1-28). Note that in $[\mathrm{MM}]$, every data has to be extended to $T_{x_{0}} X$ to make the analysis work, but as we admit the result, we do not have to worry about it and simply restrict ourselves to a neighborhood of $x_{0}$.

Now, it is clear that in the formula for $\mathscr{L}_{t}$ in (1-28), the term

$$
\mathscr{L}_{t}^{0}:=-g^{i j}(t Z)\left(\nabla_{t, e_{i}} \nabla_{t, e_{j}}-t \Gamma_{i j}^{k}(t Z) \nabla_{t, e_{k}}\right)-2 \pi n+4 \pi \mathcal{N}+t^{2} \Phi_{E}^{0}(t Z)
$$

preserves the degree, because $\Gamma^{\Lambda^{0, \bullet}}$ does (as explained in Remark 1.6). Thus, using (1-26) and taking Taylor expansion of $\mathscr{L}_{t}$ in (1-28), we can write

$$
\mathscr{L}_{t}^{0}=\mathscr{L}_{0}+\sum_{r=1}^{\infty} t^{r} \mathcal{O}_{r}^{0}, \quad t^{2} \Phi_{E}^{ \pm 2}(t Z)=\sum_{r=2}^{\infty} t^{r} \mathcal{O}_{r}^{ \pm 2} .
$$

From (1-30), we get (1-24).

Finally, due to the presence of the conjugation by $\kappa^{1 / 2}$ in (1-22), $\mathscr{L}_{t}$ is a formally self-adjoint operator on $\mathscr{C}^{\infty}\left(\mathbb{R}^{2 n}, \mathrm{E}_{x_{0}}\right)$ with respect to $\|\cdot\|_{L^{2}}$. So are $\mathscr{L}_{0}$ and $\mathcal{O}_{r}$.

Recall that $\mathscr{R}=\left(R^{E}\right)^{0,2} \in \Omega^{0,2}(X, \operatorname{End}(E))$.

Proposition 1.9. We have

$$
\mathcal{O}_{1}=0
$$

For $\mathrm{O}_{2}$, we have the formulas

$$
\mathcal{O}_{2}^{+2}=\mathscr{R}_{x_{0}}, \quad \mathcal{O}_{2}^{-2}=\left(\mathscr{R}_{x_{0}}\right)^{*}
$$

and

$$
\begin{aligned}
\mathcal{O}_{2}^{0}=\frac{1}{3}\left\langle R_{x_{0}}^{T X}\right. & \left.\left(Z, e_{i}\right) Z, e_{j}\right\rangle \nabla_{0, e_{i}} \nabla_{0, e_{j}}-R_{x_{0}}^{E}\left(w_{j}, \bar{w}_{j}\right)-\frac{1}{6} r_{x_{0}}^{X} \\
& +\left(\left\langle\frac{1}{3} R_{x_{0}}^{T X}\left(Z, e_{k}\right) e_{k}+\frac{\pi}{3} R_{x_{0}}^{T X}(z, \bar{z}) Z, e_{j}\right\rangle-R_{x_{0}}^{E}\left(Z, e_{j}\right)\right) \nabla_{0, e_{j}} .
\end{aligned}
$$

Proof. For $F=L, E$ or $\Lambda^{0, \bullet}\left(T^{*} X\right)$, it is known that (see, for instance, [MM, Lemma 1.2.4])

$$
\sum_{|\alpha|=r}\left(\partial^{\alpha} \Gamma^{F}\right)_{x_{0}}\left(e_{j}\right) \frac{Z^{\alpha}}{\alpha !}=\frac{1}{r+1} \sum_{|\alpha|=r-1}\left(\partial^{\alpha} R^{F}\right)_{x_{0}}\left(Z, e_{j}\right) \frac{Z^{\alpha}}{\alpha !},
$$


and in particular,

$$
\Gamma_{Z}^{F}\left(e_{j}\right)=\frac{1}{2} R_{x_{0}}^{F}\left(Z, e_{j}\right)+O\left(|Z|^{2}\right) .
$$

Furthermore, we know from the Gauss lemma (see, e.g., [MM, Equation (1.2.19)]) that

$$
g_{i j}(Z)=\delta_{i j}+O\left(|Z|^{2}\right)
$$

This implies that

$$
\kappa(Z)=\left|\operatorname{det}\left(g_{i j}(Z)\right)\right|^{1 / 2}=1+O\left(|Z|^{2}\right) .
$$

Moreover, the second line of [MM, Equation (4.1.103)] entails

$$
\frac{\sqrt{-1}}{2 \pi} R_{Z}^{L}\left(Z, e_{j}\right)=\left\langle J Z, e_{j}\right\rangle+O\left(|Z|^{3}\right),
$$

and thus by (1-34) and (1-38),

$$
\Gamma_{Z}^{L}=\frac{1}{2} R_{x_{0}}^{L}\left(Z, e_{j}\right)+O\left(|Z|^{3}\right) .
$$

Using (1-28), (1-35), (1-37) and (1-39), we see that

$$
\nabla_{t}=\nabla_{0}+O\left(t^{2}\right) .
$$

Finally, using again (1-28), (1-36) and (1-40), we get $\mathcal{O}_{1}=0$.

Concerning $\mathcal{O}_{2}^{ \pm 2}$, from (1-30), we see that

$$
\begin{aligned}
& \mathcal{O}_{2}^{+2}=\Phi_{E}^{+2}(0)=R_{x_{0}}^{E}\left(\bar{w}_{\ell}, \bar{w}_{m}\right) \bar{w}^{\ell} \wedge \bar{w}^{m}=\left(R_{x_{0}}^{E}\right)^{0,2}=\mathscr{R}_{x_{0}}, \\
& \mathcal{O}_{2}^{-2}=\Phi_{E}^{-2}(0)=R_{x_{0}}^{E}\left(w_{\ell}, w_{m}\right) i_{\bar{w}_{\ell}} i_{\bar{w}_{m}}=\left(\left(R_{x_{0}}^{E}\right)^{0,2}\right)^{*}=\left(\mathscr{R}_{x_{0}}\right)^{*} .
\end{aligned}
$$

Finally, by (1-29) and [MM, Equation (4.1.34)], we see that our $\mathscr{L}_{t}^{0}$ corresponds to $\mathscr{L}_{t}$ in [MM]. Thus, by (1-30) and [MM, Equation (4.1.31)], our $\mathcal{O}_{2}^{0}$ is equal to their $\mathcal{O}_{2}$ (this is because in their case, $E$ is holomorphic, so $R^{E}$ is a $(1,1)$-form and there is no term changing the degree in $\left(\bar{\partial}^{L^{p} \otimes E}+\bar{\partial}^{L^{p} \otimes E, *}\right)^{2}$; but the terms preserving the degree are the same as ours). Hence (1-33) follows from [MM, Theorem 4.1.25].

1C. Bergman kernel of the limit operator $\mathscr{L}_{0}$. In this subsection, we study more precisely the operator $\mathscr{L}_{0}$.

We introduce the complex coordinates $z=\left(z_{1}, \ldots, z_{n}\right)$ on $\mathbb{C}^{n} \simeq \mathbb{R}^{2 n}$. Thus, we get $Z=z+\bar{z}, w_{j}=\sqrt{2} \partial / \partial z_{j}$ and $\bar{w}_{j}=\sqrt{2} \partial / \partial \bar{z}_{j}$. We will identify $z$ to $\sum_{j} z_{j} \partial / \partial z_{j}$
and $\bar{z}$ to $\sum \bar{z}_{j} \partial / \partial \bar{z}_{j}$ when we consider $z$ and $\bar{z}$ as vector fields.

and $\bar{z}$ to $\sum_{j} \bar{z}_{j} \partial / \partial \bar{z}_{j}$ when we consider $z$ and $\bar{z}$ as vector fields.
Set

$$
b_{j}=-2 \nabla_{0, \partial / \partial z_{j}}, \quad b_{j}^{+}=2 \nabla_{0, \partial / \partial \bar{z}_{j}},
$$

$$
b=\left(b_{1}, \ldots, b_{n}\right), \quad \mathscr{L}=-\sum_{i}\left(\nabla_{0, e_{i}}\right)^{2}-2 \pi n .
$$


By definition, $\nabla_{0}=\nabla+\frac{1}{2} R_{x_{0}}^{L}(Z, \cdot)$ so we get

$$
b_{i}=-2 \frac{\partial}{\partial z_{i}}+\pi \bar{z}_{i}, \quad b_{i}^{+}=2 \frac{\partial}{\partial \bar{z}_{i}}+\pi z_{i},
$$

and for any polynomial $g(z, \bar{z})$ in $z$ and $\bar{z}$,

$$
\begin{aligned}
{\left[b_{i}, b_{j}^{+}\right] } & =-4 \pi \delta_{i j}, \quad\left[b_{i}, b_{j}\right]=\left[b_{i}^{+}, b_{j}^{+}\right]=0, \\
{\left[g(z, \bar{z}), b_{j}\right] } & =2 \frac{\partial}{\partial z_{j}} g(z, \bar{z}), \quad\left[g(z, \bar{z}), b_{j}^{+}\right]=-2 \frac{\partial}{\partial \bar{z}_{j}} g(z, \bar{z}) .
\end{aligned}
$$

Finally, a simple calculation shows

$$
\mathscr{L}=\sum_{i} b_{i} b_{i}^{+} \quad \text { and } \quad \mathscr{L}_{0}=\mathscr{L}+4 \pi \mathcal{N}
$$

Recall that we denoted by $\|\cdot\|_{L^{2}}$ the $L^{2}$-norm associated with $h^{\mathbb{E}_{x_{0}}}$ and $d v_{T X}$. For this form we have $b_{i}^{+}=\left(b_{i}\right)^{*}$, therefore $\mathscr{L}$ and $\mathscr{L}_{0}$ are self-adjoint.

The next theorem is proved in [MM, Theorem 4.1.20]:

Theorem 1.10. The spectrum of the restriction of $\mathscr{L}$ to $L^{2}\left(\mathbb{R}^{2 n}\right)$ is $\operatorname{Sp}\left(\left.\mathscr{L}\right|_{L^{2}\left(\mathbb{R}^{2 n}\right)}\right)=$ $4 \pi \mathbb{N}$ and an orthogonal basis of the eigenspace for the eigenvalue $4 \pi k$ is

$$
b^{\alpha}\left(z^{\beta} \exp \left(-\frac{\pi}{2}|z|^{2}\right)\right) \quad \text { with } \alpha, \beta \in \mathbb{N}^{n} \text { and } \sum_{i} \alpha_{i}=k .
$$

In particular, an orthonormal basis of $\operatorname{ker}\left(\left.\mathscr{L}\right|_{L^{2}\left(\mathbb{R}^{2 n}\right)}\right)$ is

$$
\left(\frac{\pi^{|\beta|}}{\beta !}\right)^{1 / 2} z^{\beta} \exp \left(-\frac{\pi}{2}|z|^{2}\right)
$$

and thus if $\mathscr{P}\left(Z, Z^{\prime}\right)$ is the smooth kernel of $\mathscr{P}$, the orthogonal projection from $\left(L^{2}\left(\mathbb{R}^{2 n}\right),\|\cdot\|_{0}\right)$ onto $\operatorname{ker}(\mathscr{L})$ (where $\|\cdot\|_{0}$ is the $L^{2}$-norm associated to $g_{x_{0}}^{T X}$ ) with respect to $d v_{T X}\left(Z^{\prime}\right)$, we have

$$
\mathscr{P}\left(Z, Z^{\prime}\right)=\exp \left(-\frac{\pi}{2}\left(|z|^{2}+\left|z^{\prime}\right|^{2}-2 z \cdot \bar{z}^{\prime}\right)\right) .
$$

Now let $P^{N}$ be the orthogonal projection from $\left(L^{2}\left(\mathbb{R}^{2 n}, \mathbb{E}_{x_{0}}\right),\|\cdot\|_{L^{2}}\right)$ onto $N:=$ $\operatorname{ker}\left(\mathscr{L}_{0}\right)$, and $P^{N}\left(Z, Z^{\prime}\right)$ be its smooth kernel with respect to $d v_{T X}\left(Z^{\prime}\right)$. From (1-45), we have

$$
P^{N}\left(Z, Z^{\prime}\right)=\mathscr{P}\left(Z, Z^{\prime}\right) I_{0}
$$




\section{The first coefficient in the asymptotic expansion}

In this section we prove Theorem 0.4. We will proceed as follows. In Section 2A, following [MM, Section 4.1.7], we will give a formula for $\boldsymbol{b}_{r}$ involving the $\mathcal{O}_{k}$ and $\mathscr{L}_{0}$. In Section 2B, we will see how this formula entails Theorem 0.4.

2A. A formula for $\boldsymbol{b}_{\boldsymbol{r}}$. By Theorem 1.10 and (1-45), we know that for every $\lambda \in \delta$ the unit circle in $\mathbb{C},\left(\lambda-\mathscr{L}_{0}\right)^{-1}$ exists.

Let $f(\lambda, t)$ be a formal power series on $t$ with values in $\operatorname{End}\left(L^{2}\left(\mathbb{R}^{2 n}, \mathbb{E}_{x_{0}}\right)\right)$ :

$$
f(\lambda, t)=\sum_{r=0}^{+\infty} t^{r} f_{r}(\lambda) \quad \text { with } f_{r}(\lambda) \in \operatorname{End}\left(L^{2}\left(\mathbb{R}^{2 n}, \mathbb{E}_{x_{0}}\right)\right) .
$$

Consider the equation of formal power series on $t$ for $\lambda \in \delta$,

$$
\left(\lambda-\mathscr{L}_{0}-\sum_{r=1}^{+\infty} t^{r} \mathcal{O}_{r}\right) f(\lambda, t)=\operatorname{Id}_{L^{2}\left(\mathbb{R}^{2 n}, \mathbb{E}_{x_{0}}\right)} .
$$

We then find that

$$
\begin{aligned}
& f_{0}(\lambda)=\left(\lambda-\mathscr{L}_{0}\right)^{-1}, \\
& f_{r}(\lambda)=\left(\lambda-\mathscr{L}_{0}\right)^{-1} \sum_{j=1}^{r} \mathcal{O}_{j} f_{r-j}(\lambda) .
\end{aligned}
$$

Thus by (1-31) and by induction,

$$
f_{r}(\lambda)=\left(\sum_{\substack{r_{1}+\ldots+r_{k}=r \\ r_{j} \geq 2}}\left(\lambda-\mathscr{L}_{0}\right)^{-1} \mathcal{O}_{r_{1}} \cdots\left(\lambda-\mathscr{L}_{0}\right)^{-1} \mathcal{O}_{r_{k}}\right)\left(\lambda-\mathscr{L}_{0}\right)^{-1}
$$

Definition 2.1. Following [MM, Equation (4.1.91)], we define $\mathscr{F}_{r}$ by

$$
\mathscr{F}_{r}=\frac{1}{2 \pi \sqrt{-1}} \int_{\delta} f_{r}(\lambda) d \lambda
$$

and we denote by $\mathscr{F}_{r}\left(Z, Z^{\prime}\right)$ its smooth kernel with respect to $d v_{T X}\left(Z^{\prime}\right)$.

Theorem 2.2. The following equation holds:

$$
\boldsymbol{b}_{r}\left(x_{0}\right)=\mathscr{F}_{2 r}(0,0) .
$$

Proof. This formula follows from [MM, Theorem 8.1.4] as [MM, Equation (4.1.97)] follows from [MM, Theorem 4.1.24], remembering that in our situation the Bergman kernel $P_{p}$ is not supported in degree 0 . 
2B. Proof of Theorem 0.4. Let $T_{\boldsymbol{r}}(\lambda)=\left(\lambda-\mathscr{L}_{0}\right)^{-1} \mathcal{O}_{r_{1}} \cdots\left(\lambda-\mathscr{L}_{0}\right)^{-1} \mathcal{O}_{r_{k}}\left(\lambda-\mathscr{L}_{0}\right)^{-1}$ be the term in the sum (2-4) corresponding to $r=\left(r_{1}, \ldots, r_{k}\right)$. Let $N^{\perp}$ be the orthogonal of $N$ in $L^{2}\left(\mathbb{R}^{2 n}, \mathbb{E}_{x_{0}}\right)$, and $P^{N^{\perp}}$ the associated orthogonal projector. In $T_{\boldsymbol{r}}(\lambda)$, each term $\left(\lambda-\mathscr{L}_{0}\right)^{-1}$ can be decomposed as

$$
\left(\lambda-\mathscr{L}_{0}\right)^{-1}=\left(\lambda-\mathscr{L}_{0}\right)^{-1} P^{N^{\perp}}+\frac{1}{\lambda} P^{N} .
$$

Set

$$
L^{N^{\perp}}(\lambda)=\left(\lambda-\mathscr{L}_{0}\right)^{-1} P^{N^{\perp}}, \quad L^{N}(\lambda)=\frac{1}{\lambda} P^{N}
$$

By (1-45), $\mathscr{L}_{0}$ preserves the degree, and thus so do $\left(\lambda-\mathscr{L}_{0}\right)^{-1}, L^{N^{\perp}}$ and $L^{N}$.

For $\eta=\left(\eta_{1}, \ldots, \eta_{k+1}\right) \in\left\{N, N^{\perp}\right\}^{k+1}$, let

$$
T_{\boldsymbol{r}}^{\eta}(\lambda)=L^{\eta_{1}}(\lambda) \mathcal{O}_{r_{1}} \cdots L^{\eta_{k}}(\lambda) \mathcal{O}_{r_{k}} L^{\eta_{k+1}}(\lambda) .
$$

We can decompose

$$
T_{\boldsymbol{r}}(\lambda)=\sum_{\eta=\left(\eta_{1}, \ldots, \eta_{k+1}\right)} T_{r}^{\eta}(\lambda)
$$

and by (2-4) and (2-5),

$$
\mathscr{F}_{2 r}=\frac{1}{2 \pi \sqrt{-1}} \sum_{\substack{r_{1}+\cdots+r_{k}=2 r \\\left(\eta_{1}, \ldots, \eta_{k+1}\right)}} \int_{\delta} T_{r}^{\eta}(\lambda) d \lambda .
$$

Note that $L^{N^{\perp}}(\lambda)$ is an holomorphic function of $\lambda$, so

$$
\int_{\delta} L^{N^{\perp}}(\lambda) \mathcal{O}_{r_{1}} \cdots L^{N^{\perp}}(\lambda) \mathcal{O}_{r_{k}} L^{N^{\perp}}(\lambda) d \lambda=0 .
$$

Thus, in (2-11), every nonzero term that appears contains at least one $L^{N}(\lambda)$,

$$
\int_{\delta} T_{r}^{\eta}(\lambda) d \lambda \neq 0 \quad \Rightarrow \quad \text { there exists an } i_{0} \text { such that } \eta_{i_{0}}=N
$$

Now fix $k$ and $j$ in $\mathbb{N}$. Let $s \in L^{2}\left(\mathbb{R}^{2 n}, \mathbb{E}_{x_{0}}\right)$ be a form of degree $2 j, r \in(\mathbb{N} \backslash\{0,1\})^{k}$ such that $\sum_{i} r_{i}=2 r$ and $\eta=\left(\eta_{1}, \ldots, \eta_{k+1}\right) \in\left\{N, N^{\perp}\right\}^{k+1}$ such that there is an $i_{0}$ satisfying $\eta_{i_{0}}=N$. We want to find a necessary condition for $I_{2 j} T_{r}^{\eta}(\lambda) I_{2 j} s$ to be nonzero.

Suppose then that $I_{2 j} T_{\boldsymbol{r}}^{\eta}(\lambda) I_{2 j} s \neq 0$. Since $L^{\eta_{i}}=\frac{1}{\lambda} P^{N}$, and $N$ is concentrated in degree 0 , we must have

$$
\operatorname{deg}\left(\mathcal{O}_{r_{i_{0}}} L^{\eta_{i_{0}+1}}(\lambda) \mathcal{O}_{r_{i_{0}+1}} \cdots L^{\eta_{k}}(\lambda) \mathcal{O}_{r_{k}} L^{\eta_{k+1}}(\lambda) I_{2 j} s\right)=0
$$


but each $L^{\eta_{i}}(\lambda)$ preserves the degree, and by Theorem 1.8 each $\mathcal{O}_{r_{i}}$ lowers the degree at most by 2 , so

$$
0=\operatorname{deg}\left(\mathcal{O}_{r_{i_{0}}} L^{\eta_{i_{0}+1}}(\lambda) \mathcal{O}_{r_{i_{0}+1}} \cdots L^{\eta_{k}}(\lambda) \mathcal{O}_{r_{k}} L^{\eta_{k+1}}(\lambda) I_{2 j} s\right) \geq 2 j-2\left(k-i_{0}+1\right),
$$

and thus

$$
2 j \leq 2\left(k-i_{0}+1\right) .
$$

Similarly, $L^{\eta_{1}}(\lambda) \mathcal{O}_{r_{1}} \cdots L^{\eta_{k}}(\lambda) \mathcal{O}_{r_{k}} L^{\eta_{k+1}}(\lambda) I_{2 j} s$ must have a nonzero component in degree $2 j$ and by Theorem 1.8 each $\mathcal{O}_{r_{i}}$ rises the degree at most by 2 , so $2 j$ must be less than or equal to the number of $\mathcal{O}_{r_{i}}$ appearing before $\mathcal{O}_{r_{i}}$, that is,

$$
2 j \leq 2\left(i_{0}-1\right) \text {. }
$$

With (2-14) and (2-15), we find

$$
4 j \leq 2 k \text {. }
$$

Finally, since for every $i, r_{i} \geq 2$ and $\sum_{i=1}^{k} r_{i}=2 r$, we have $2 k \leq 2 r$, and thus

$$
4 j \leq 2 k \leq 2 r \text {. }
$$

Consequently, if $r<2 j$ we have $I_{2 j} T_{r}^{\eta}(\lambda) I_{2 j}=0$, and by (2-11), we find $I_{2 j} \mathscr{F}_{2 r} I_{2 j}=0$. Using Theorem 2.2, we find

$$
I_{2 j} \boldsymbol{b}_{r} I_{2 j}=0
$$

which, combined with Theorem 0.3, entails the first part of Theorem 0.4.

For the second part of this theorem, let us focus on the case $r=2 j$. We also suppose that $j \geq 1$, because in the case $j=0$, [MM, Equation (8.1.5)] implies that $\boldsymbol{b}_{0}\left(x_{0}\right)=\mathscr{F}_{0}(0,0)=I_{0} \mathscr{P}(0,0)=I_{0}$, so Theorem 0.4 is true for $j=0$.

In $I_{2 j} \mathscr{F}_{4 j} I_{2 j}$, there is only one term satisfying equations (2-14), (2-15) and (2-17). First we see that (2-17) implies that $r=k=2 j$ and for all $i, r_{i}=2$, while (2-14) and (2-15) imply that the $i_{0}$ such that $\eta_{i_{0}}=N$ is unique and equal to $j$. Moreover, only $\mathcal{O}_{2}^{+2}$ and $\mathcal{O}_{2}^{-2}$ appear in $I_{2 j} \mathscr{F}_{4 j} I_{2 j}$, not $\mathcal{O}_{2}^{0}$, because the degree must decrease by $2 j$ and then increase by $2 j$ with $k=2 j \mathcal{O}_{r_{i}}$ available. To summarize,

$$
\begin{aligned}
I_{2 j} \mathscr{F}_{4 j} I_{2 j}= & \frac{1}{2 \pi \sqrt{-1}} \int_{\delta}\left(I_{2 j}\left(\left(\lambda-\mathscr{L}_{0}\right)^{-1} P^{N^{\perp}} \mathcal{O}_{2}^{+2}\right)^{j}\right. \\
& \left.\times \frac{1}{\lambda} P^{N}\left(\mathcal{O}_{2}^{-2}\left(\lambda-\mathscr{L}_{0}\right)^{-1} P^{N^{\perp}}\right)^{j} I_{2 j}\right) d \lambda \\
= & I_{2 j}\left(\mathscr{L}_{0}^{-1} P^{N^{\perp}} \mathcal{O}_{2}^{+2}\right)^{j} P^{N}\left(\mathcal{O}_{2}^{-2} \mathscr{L}_{0}^{-1} P^{N^{\perp}}\right)^{j} I_{2 j} \\
= & I_{2 j}\left(\mathscr{L}_{0}^{-1} \mathcal{O}_{2}^{+2}\right)^{j} P^{N}\left(\mathcal{O}_{2}^{-2} \mathscr{L}_{0}{ }^{-1}\right)^{j} I_{2 j} .
\end{aligned}
$$

Because by (1-45), $L^{2}\left(\mathbb{R}^{2 n},\left(\Lambda^{0,>0}\left(T^{*} X\right) \otimes E\right)_{x_{0}}\right) \subset N^{\perp}$, we removed the $P^{N^{\perp}}$ in the last line. 
Let $A=I_{2 j}\left(\mathscr{L}_{0}^{-1} \mathcal{O}_{2}^{+2}\right)^{j} P^{N}$. Since $\left(\mathcal{O}_{2}^{+2}\right)^{*}=\mathcal{O}_{2}^{-2}$ (see Proposition 1.9) and $\mathscr{L}_{0}$ is self-adjoint, the adjoint of $A$ is $A^{*}=P^{N}\left(\mathcal{O}_{2}^{-2} \mathscr{L}_{0}^{-1}\right)^{j} I_{2 j}$, and thus

$$
I_{2 j} \mathscr{F}_{4 j} I_{2 j}=A A^{*} .
$$

Recall that $P^{N}=\mathscr{P} I_{0}$ (see (1-49)). Let $s \in L^{2}\left(\mathbb{R}^{2 n}, E_{x_{0}}\right) ;$ since $\mathscr{L}_{0}=\mathscr{L}+4 \pi \mathcal{N}$ and $\mathscr{L} \mathscr{P}_{S}=0,\left(\mathscr{P}_{S}\right) \mathscr{R}_{x_{0}}$ is an eigenfunction of $\mathscr{L}_{0}$ for the eigenvalue $2 \times 4 \pi$. Thus,

$$
\mathscr{L}_{0}^{-1} \mathcal{O}_{2}^{+2} P^{N}{ }_{s}=\mathscr{L}_{0}^{-1} \mathcal{O}_{2}^{+2} \mathscr{P}_{S}=\mathscr{L}_{0}^{-1}\left(\left(\mathscr{P}_{S}\right) \mathscr{R}_{x_{0}}\right)=\frac{1}{4 \pi} \frac{1}{2} \mathscr{\Re}_{x_{0}} \mathscr{P}_{S} .
$$

Now, an easy induction shows that

$$
A=\frac{1}{(4 \pi)^{j}} \frac{1}{2 \times 4 \times \cdots \times 2 j} I_{2 j} \mathscr{R}_{x_{0}}^{j} \mathscr{P}=\frac{1}{(4 \pi)^{j}} \frac{1}{2^{j} j !} I_{2 j} \mathscr{R}_{x_{0}}^{j} \mathscr{P} .
$$

Let $A\left(Z, Z^{\prime}\right)$ and $A^{*}\left(Z, Z^{\prime}\right)$ be the smooth kernels of $A$ and $A^{*}$ with respect to $d v_{T X}\left(Z^{\prime}\right)$. By (2-19), $I_{2 j} \mathscr{F}_{4 j} I_{2 j}(0,0)=\int_{\mathbb{R}^{2 n}} A(0, Z) A^{*}(Z, 0) d Z$. Thanks to

$$
\int_{\mathbb{R}^{2 n}} \mathscr{P}(0, Z) \mathscr{P}(Z, 0) d Z=(\mathscr{P} \circ \mathscr{P})(0,0)=\mathscr{P}(0,0)=1
$$

and (2-21), we find (0-12).

\section{The second coefficient in the asymptotic expansion}

In this section, we prove Theorem 0.7. Using (2-6), we know that

$$
I_{2 j} \boldsymbol{b}_{2 j+1} I_{2 j}(0,0)=I_{2 j} \mathscr{F}_{4 j+2} I_{2 j}(0,0) \text {. }
$$

In Section 3A, we decompose this into three terms, and then in Sections 3B and 3C we handle them separately.

Fix $j \in \llbracket 0, n \rrbracket$. For every smoothing operator $F$ acting on $L^{2}\left(\mathbb{R}^{2 n}, \mathbb{E}_{x_{0}}\right)$ in this section, we denote by $F\left(Z, Z^{\prime}\right)$ its smooth kernel with respect to $d v_{T X}\left(Z^{\prime}\right)$.

3A. Decomposition of the problem. Applying inequality (2-17) with $r=2 j+1$, we see that in $I_{2 j} \mathscr{F}_{4 j+2} I_{2 j}$, the nonzero terms $\int_{\delta} T_{\boldsymbol{r}}^{\eta}(\lambda) d \lambda$ in the decomposition (2-11) satisfy $k=2 j$ or $k=2 j+1$. Since $\sum_{i} r_{i}=4 j+2$ and $r_{i} \geq 2$, we see that in $I_{2 j} \mathscr{F}_{4 j+2} I_{2 j}$ there are three types of terms $T_{\mathrm{r}}^{\eta}(\lambda)$ with nonzero integral, in which

- for $k=2 j$,

- there are $2 j-2 \mathcal{O}_{r_{i}}$ equal to $\mathcal{O}_{2}$ and 2 equal to $\mathcal{O}_{3}$ and we denote by I the sum of these terms,

- there are $2 j-1 \mathcal{O}_{r_{i}}$ equal to $\mathcal{O}_{2}$ and 1 equal to $\mathcal{O}_{4}$ and we denote by II the sum of these terms,

- for $k=2 j+1$,

- all the $\mathcal{O}_{r_{i}}$ are equal to $\mathcal{O}_{2}$ and we denote by III the sum of these terms. 
We thus have a decomposition

$$
I_{2 j} \mathscr{F}_{4 j+2} I_{2 j}=\mathrm{I}+\mathrm{II}+\mathrm{III} .
$$

Remark 3.1. Note that for I and II to be nonzero, we must have $j \geq 1$. Moreover, in the first two cases, as $k=2 j$, by the same reasoning as in Section 2B, (2-14) and (2-15) imply that the $i_{0}$ such that $\eta_{i_{0}}=N$ is unique and equal to $j$, and that only $\mathcal{O}_{2}^{ \pm 2}, \mathcal{O}_{3}^{ \pm 2}$ and $\mathcal{O}_{4}^{ \pm 2}$ appear in I and II, not some $\mathcal{O}_{r_{i}}^{0}$.

\section{B. The term involving only $\mathrm{O}_{2}$.}

Lemma 3.2. In any term $T_{\mathrm{r}}^{\eta}(\lambda)$ appearing in the sum III (with nonvanishing integral), the $i_{0}$ such that $\eta_{i_{0}}=N$ is unique and equal to $j$ or $j+1$. If we denote by $\mathrm{III}_{a}$ and $\mathrm{III}_{b}$ the sums corresponding to these two cases, we have

$$
\begin{aligned}
& \mathrm{III}_{a}=\sum_{k=0}^{j} I_{2 j}\left(\mathscr{L}_{0}^{-1} \mathcal{O}_{2}^{+2}\right)^{j-k}\left(\mathscr{L}_{0}^{-1} \mathcal{O}_{2}^{0}\right)\left(\mathscr{L}_{0}^{-1} \mathcal{O}_{2}^{+2}\right)^{k} P^{N}\left(\mathcal{O}_{2}^{-2} \mathscr{L}_{0}^{-1}\right)^{j} I_{2 j}, \\
& \mathrm{III}_{b}=\left(\mathrm{III}_{a}\right)^{*} \\
& \mathrm{III}=\mathrm{III}_{a}+\mathrm{III}_{b} .
\end{aligned}
$$

Remark 3.3. For the same reason as for (2-18), we have removed the $P^{N^{\perp}}$ in (3-3) without getting any problem concerning the existence of $\mathscr{L}_{0}^{-1}$.

Proof. Fix a term $T_{\mathrm{r}}^{\eta}(\lambda)$ appearing in the sum III with nonvanishing integral. Using again the same reasoning as in Section 2B, we see that there exists at most two indices $i_{0}$ such that $\eta_{i_{0}}=N$, and that they are in $\{j, j+1\}$. Indeed, with only $2 j+1$ $\mathcal{O}_{r_{i}}$ at our disposal, we need $j$ of them before the first $P^{N}$, and $j$ after the last one.

Now, the only possible term with $\eta_{j}=\eta_{j+1}=N$ is

$$
\left(\mathscr{L}_{0}^{-1} \mathcal{O}_{2}^{+2}\right)^{j} P^{N} \mathcal{O}_{2}^{0} P^{N}\left(\mathcal{O}_{2}^{-2} \mathscr{L}_{0}^{-1}\right)^{j}
$$

To prove that this term is vanishing, using (1-33), [Ma and Marinescu 2012, Equations (3.13), (3.16b) and 4.1a], we see that $\mathscr{P} \mathcal{O}_{2}^{0} \mathscr{P}=0$, and so

$$
P^{N} \mathcal{O}_{2}^{0} P^{N}=\mathscr{P} \mathcal{O}_{2}^{0} \mathscr{P} I_{0}=0 .
$$

We have proved the first part of the lemma.

The second part follows from the reasoning made at the beginning of this proof, and the facts that $i_{0}$ is unique, $\mathcal{O}_{2}^{0}$ is self-adjoint and $\left(\mathscr{L}_{0}^{-1} \mathcal{O}_{2}^{+2}\right)^{*}=\mathcal{O}_{2}^{-2} \mathscr{L}_{0}{ }^{-1}$.

Let us compute the term that appears in (3-3),

$$
\operatorname{III}_{a, k}:=I_{2 j}\left(\mathscr{L}_{0}^{-1} \mathcal{O}_{2}^{+2}\right)^{j-k}\left(\mathscr{L}_{0}^{-1} \mathcal{O}_{2}^{0}\right)\left(\mathscr{L}_{0}^{-1} \mathcal{O}_{2}^{+2}\right)^{k} P^{N}\left(\mathcal{O}_{2}^{-2} \mathscr{L}_{0}^{-1}\right)^{j} I_{2 j} .
$$


With (2-21), we know that

$$
P^{N}\left(\mathcal{O}_{2}^{-2} \mathscr{L}_{0}^{-1}\right)^{j} I_{2 j}=\frac{1}{(4 \pi)^{j}} \frac{1}{2^{j} j !} \mathscr{P}\left(\Re_{x_{0}}^{j}\right)^{*} I_{2 j},
$$

and

$$
\begin{aligned}
I_{2 j}\left(\mathscr{L}_{0}^{-1} \mathcal{O}_{2}^{+2}\right)^{j-k}\left(\mathscr{L}_{0}^{-1} \mathcal{O}_{2}^{0}\right)\left(\mathscr{L}_{0}^{-1} \mathcal{O}_{2}^{+2}\right)^{k} P^{N} \\
=\frac{1}{(4 \pi)^{k}} \frac{1}{2^{k} k !} I_{2 j}\left(\mathscr{L}_{0}^{-1} \mathcal{O}_{2}^{+2}\right)^{j-k} \mathscr{L}_{0}{ }^{-1}\left(\mathcal{O}_{2}^{0} \mathscr{R}_{x_{0}}^{k} \mathscr{P}\right) I_{0}
\end{aligned}
$$

Let

$$
R_{k \bar{m} \ell \bar{q}}=\left\langle R^{T X}\left(\frac{\partial}{\partial z_{k}}, \frac{\partial}{\partial \bar{z}_{m}}\right) \frac{\partial}{\partial z_{\ell}}, \frac{\partial}{\partial \bar{z}_{q}}\right\rangle_{x_{0}} \quad \text { and } \quad R_{k \bar{\ell}}^{E}=R_{x_{0}}^{E}\left(\frac{\partial}{\partial z_{k}}, \frac{\partial}{\partial \bar{z}_{\ell}}\right) .
$$

By [ibid., Lemma 3.1], we know that

$$
R_{k \bar{m} \ell \bar{q}}=R_{\ell \bar{m} k \bar{q}}=R_{k \bar{q} \ell \bar{m}}=R_{\ell \bar{q} k \bar{m}} \quad \text { and } \quad r_{x_{0}}^{X}=8 R_{m \bar{m} q \bar{q}} .
$$

Once again, our $\mathcal{O}_{2}^{0}$ correspond to the $\mathcal{O}_{2}$ of [Ma and Marinescu 2012] (see (1-33) and [ibid., Equations (3.13), (3.16b)]), so we can use [ibid., Equation (4.6)] to get

$$
\begin{aligned}
\mathcal{O}_{2}^{0} \mathscr{R}_{x_{0}}^{k} \mathscr{P}=\left(\frac{1}{6} b_{m} b_{q} R_{k \bar{m} \ell \bar{q}} z_{k} z_{\ell}+\right. & \frac{4}{3} b_{q} R_{\ell \bar{k} k \bar{q}} z_{\ell} \\
& \left.-\frac{1}{3} \pi b_{q} R_{k \bar{m} \ell \bar{q}} z_{k} z_{\ell} \bar{z}_{m}^{\prime}+b_{q} R_{\ell \bar{q}}^{E} z_{\ell}\right) \mathscr{R}_{x_{0}}^{k} \mathscr{P},
\end{aligned}
$$

Set

$$
\begin{aligned}
& a=\frac{1}{6} b_{m} b_{q} R_{k \bar{m} \ell \bar{q}} z_{k} z_{\ell}, \quad b=\frac{4}{3} b_{q} R_{\ell \bar{k} k \bar{q}} z_{\ell}, \\
& c=-\frac{1}{3} \pi b_{q} R_{k \bar{m} \ell \bar{q}} z_{k} z_{\ell} \bar{z}_{m}^{\prime}, \quad d=b_{q} R_{\ell \bar{q}}^{E} z_{\ell} .
\end{aligned}
$$

Thanks to (1-45), (1-46) and (3-11), we find

$$
\mathscr{L}_{0}^{-1} \mathcal{O}_{2}^{0} \mathscr{R}_{x_{0}}^{k} \mathscr{P} I_{0}=\left(\frac{a}{4 \pi(2+2 k)}+\frac{b+c+d}{4 \pi(1+2 k)}\right) \Re_{x_{0}}^{k} \mathscr{P} I_{0},
$$

and by induction, (3-8) becomes

$$
=\frac{1}{(4 \pi)^{j+1}} \frac{1}{2^{k} k !} I_{2 j} \mathscr{R}_{x_{0}}^{j-k}\left(\frac{a}{(2+2 k) \cdots(2+2 j)}+\frac{b+c+d}{(1+2 k) \cdots(1+2 j)}\right) \mathscr{R}_{x_{0}}^{k} \mathscr{P} I_{0} .
$$

Lemma 3.4. We have

$$
\begin{aligned}
& \left(a \mathscr{R}_{x_{0}}^{k} \mathscr{P}\right)(0, Z)=\frac{1}{6} r_{x_{0}}^{X} \mathscr{R}_{x_{0}}^{k} \mathscr{P}(0, Z), \quad\left(b \mathscr{R}_{x_{0}}^{k} \mathscr{P}\right)(0, Z)=-\frac{1}{3} r_{x_{0}}^{X} \mathscr{R}_{x_{0}}^{k} \mathscr{P}(0, Z), \\
& \left(c \mathscr{R}_{x_{0}}^{k} \mathscr{P}\right)(0, Z)=0, \quad\left(d \Re_{x_{0}}^{k} \mathscr{P}\right)(0, Z)=-2 R_{q \bar{q}}^{E} \mathscr{R}_{x_{0}}^{k} \mathscr{P}(0, Z) .
\end{aligned}
$$


Proof. This lemma is a consequence of the relations (1-44) and (3-10). For demonstration, we will compute $\left(b \mathscr{R}_{x_{0}}^{k} \mathscr{P}\right)(0, Z)$; the other terms are similar.

$$
\begin{aligned}
\left(b \mathscr{R}_{x_{0}}^{k} \mathscr{P}\right)(0, Z) & =\left(\frac{4}{3} b_{q} R_{\ell \bar{k} k \bar{q}} z_{\ell} \mathscr{R}_{x_{0}}^{k} \mathscr{P}\right)(0, Z) \\
& =\frac{4}{3} R_{\ell \bar{k} k \bar{q}} \Re_{x_{0}}^{k}\left(\left(z_{\ell} b_{q}-2 \delta_{\ell q}\right) \mathscr{P}\right)(0, Z) \\
& =-\frac{8}{3} R_{\ell \bar{k} k \bar{\ell}} \mathscr{R}_{x_{0}}^{k} \mathscr{P}(0, Z)=-\frac{1}{3} r_{x_{0}}^{X} \mathscr{R}_{x_{0}}^{k} \mathscr{P}(0, Z) .
\end{aligned}
$$

Using (2-22), (3-6), (3-7) and (3-13), we find

$$
\begin{aligned}
\mathrm{III}_{a, k}(0,0)=I_{2 j} \mathrm{C}_{j}(j) \mathscr{R}_{x_{0}}^{j-k}\left[\frac { 1 } { 6 } \left(\mathrm{C}_{j+1}(j\right.\right. & \left.+1)-\frac{\mathrm{C}_{j}(k)}{2 \pi(2 k+1)}\right) r_{x_{0}}^{X} \\
& \left.-\frac{\mathrm{C}_{j}(k)}{2 \pi(2 k+1)} R_{q \bar{q}}^{E}\right] \mathscr{R}_{x_{0}}^{k}\left(\mathscr{R}_{x_{0}}^{j}\right)^{*} I_{2 j} .
\end{aligned}
$$

Notice that $2 R_{q \bar{q}}^{E}=R_{x_{0}}^{E}\left(\sqrt{2} \frac{\partial}{\partial z_{q}}, \sqrt{2} \frac{\partial}{\partial \bar{z}_{q}}\right)=R_{x_{0}}^{E}\left(w_{q}, \bar{w}_{q}\right)=\sqrt{-1} R_{\Lambda, x_{0}}^{E}$ by definition. Consequently,

$$
\begin{aligned}
\operatorname{III}_{a}(0,0)=I_{2 j} \mathrm{C}_{j}(j) \sum_{k=0}^{j} \mathscr{R}_{x_{0}}^{j-k} & {\left[\frac{1}{6}\left(\mathrm{C}_{j+1}(j+1)-\frac{\mathrm{C}_{j}(k)}{2 \pi(2 k+1)}\right) r_{x_{0}}^{X}\right.} \\
& \left.-\frac{\mathrm{C}_{j}(k)}{4 \pi(2 k+1)} \sqrt{-1} R_{\Lambda, x_{0}}^{E}\right] \mathscr{R}_{x_{0}}^{k}\left(\mathscr{R}_{x_{0}}^{j}\right)^{*} I_{2 j} .
\end{aligned}
$$

3C. The two other terms. In this subsection, we suppose that $j \geq 1$ (see Remark 3.1). Moreover, the existence of any $\mathscr{L}_{0}^{-1}$ in this section follows from the reasoning done in Remark 3.3, and this operator will be used without further precision.

Due to (1-30), we have

$$
\begin{aligned}
\mathcal{O}_{3}^{+2} & =\left.\frac{d}{d t} \Phi_{E_{0}}^{+2}(t Z)\right|_{t=0}=z_{i} \frac{\partial \mathscr{R}}{\partial z_{i}}(0)+\bar{z}_{i} \frac{\partial \mathscr{R}}{\partial \bar{z}_{i}}(0), \\
\mathcal{O}_{4}^{+2} & =\frac{z_{i} z_{j}}{2} \frac{\partial^{2} \mathscr{R} .}{\partial z_{i} \partial z_{j}}(0)+z_{i} \bar{z}_{j} \frac{\partial^{2} \mathscr{R} .}{\partial z_{i} \partial \bar{z}_{j}}(0)+\frac{\bar{z}_{i} \bar{z}_{j}}{2} \frac{\partial^{2} \mathscr{R} .}{\partial \bar{z}_{i} \partial \bar{z}_{j}}(0) .
\end{aligned}
$$

The sum I can be decomposed into three subsums: $\mathrm{I}_{a}, \mathrm{I}_{b}$ and $\mathrm{I}_{c}$, in which the two $\mathcal{O}_{3}$ appear respectively both at the left, on either side or both at the right of $P^{N}$ (see Remark 3.1). As usual, we have $\mathrm{I}_{c}=\left(\mathrm{I}_{a}\right)^{*}$.

In the same way, we can decompose $\mathrm{II}$ as $\mathrm{II}_{a}+\mathrm{II}_{b}$ : in $\mathrm{II}_{a}$ the $\mathcal{O}_{4}$ appears at the left of $P^{N}$, and in $\mathrm{II}_{b}$ at the right. Once again, $\mathrm{II}_{b}=\left(\mathrm{II}_{a}\right)^{*}$.

Computation of $\mathrm{I}_{b}(0,0)$. To compute $\mathrm{I}_{b}$, we first compute the kernel of

$$
A_{k}:=I_{2 j}\left(\mathscr{L}_{0}^{-1} \mathcal{O}_{2}^{+2}\right)^{j-k-1}\left(\mathscr{L}_{0}^{-1} \mathcal{O}_{3}^{+2}\right)\left(\mathscr{L}_{0}^{-1} \mathcal{O}_{2}^{+2}\right)^{k} \mathscr{P} I_{0}
$$

at $(0, Z)$. 
By (2-21) and (3-18),

(3-21) $A_{k}=I_{2 j}\left(\mathscr{L}_{0}^{-1} \mathcal{O}_{2}^{+2}\right)^{j-k-1}\left(\mathscr{L}_{0}^{-1} \mathcal{O}_{3}^{+2}\right) \frac{1}{(4 \pi)^{k}} \frac{1}{2^{k} k !} \mathscr{R}_{x_{0}}^{k} \mathscr{P} I_{0}$

$$
=\frac{1}{(4 \pi)^{k}} \frac{1}{2^{k} k !} I_{2 j}\left(\mathscr{L}_{0}^{-1} \mathcal{O}_{2}^{+2}\right)^{j-k-1} \mathscr{L}_{0}^{-1}\left[z_{i} \frac{\partial \mathscr{R}}{\partial z_{i}}(0)+\bar{z}_{i} \frac{\partial \mathscr{R}}{\partial \bar{z}_{i}}(0)\right] \mathscr{R}_{x_{0}}^{k} \mathscr{P} I_{0} .
$$

By Theorem 1.10, if $s \in N$, then $z_{i} s \in N$, so by the same calculation as in (2-21),

$$
\begin{aligned}
\frac{1}{(4 \pi)^{k}} \frac{1}{2^{k} k !}\left(I_{2 j}\left(\mathscr{L}_{0}^{-1} \mathcal{O}_{2}^{+2}\right)^{j-k-1} \mathscr{L}_{0}^{-1}\left[z_{i} \frac{\partial \mathscr{R}}{\partial z_{i}}(0)\right] \mathscr{R}_{x_{0}}^{k} \mathscr{P} I_{0}\right)(0, Z) \\
=\frac{1}{(4 \pi)^{j}} \frac{1}{2^{j} j !}\left(I_{2 j}\left[\mathscr{R}_{x_{0}}^{j-k-1} \frac{\partial \mathscr{R}}{\partial z_{i}}(0) \mathscr{R}_{x_{0}}^{k}\right] z_{i} \mathscr{P} I_{0}\right)(0, Z)=0 .
\end{aligned}
$$

Now by (1-43) and the formula (1-48), we have

$$
\left(b_{i}^{+} \mathscr{P}\right)\left(Z, Z^{\prime}\right)=0 \quad \text { and } \quad\left(b_{i} \mathscr{P}\right)\left(Z, Z^{\prime}\right)=2 \pi\left(\bar{z}_{i}-\bar{z}_{i}^{\prime}\right) \mathscr{P}\left(Z, Z^{\prime}\right)
$$

Thus,

$$
\begin{aligned}
& \frac{1}{(4 \pi)^{k}} \frac{1}{2^{k} k !}\left(I_{2 j}\left(\mathscr{L}_{0}^{-1} \mathcal{O}_{2}^{+2}\right)^{j-k-1} \mathscr{L}_{0}-1\left[\bar{z}_{i} \frac{\partial \mathscr{R} .}{\partial \bar{z}_{i}}(0)\right] \mathscr{R}_{x_{0}}^{k} \mathscr{P} I_{0}\right)\left(Z, Z^{\prime}\right) \\
&= \frac{1}{(4 \pi)^{k}} \frac{1}{2^{k} k !}\left(I_{2 j}\left(\mathscr{L}_{0}^{-1} \mathcal{O}_{2}^{+2}\right)^{j-k-1} \mathscr{L}_{0}^{-1}\left[\frac{\partial \mathscr{R} .}{\partial \bar{z}_{i}}(0) \mathscr{R}_{x_{0}}^{k}\right]\left(\frac{b_{i}}{2 \pi}+\bar{z}_{i}^{\prime}\right) \mathscr{P} I_{0}\right)\left(Z, Z^{\prime}\right) \\
&= \frac{1}{(4 \pi)^{k}} \frac{1}{2^{k} k !}\left(I_{2 j}\left(\mathscr{L}_{0}^{-1} \mathcal{O}_{2}^{+2}\right)^{j-k-1}\left[\frac{\partial \mathscr{R} .}{\partial \bar{z}_{i}}(0) \mathscr{R}_{x_{0}}^{k}\right]\right. \\
&= \frac{1}{(4 \pi)^{j}} \frac{1}{2^{j} j !}\left(I_{2 j}\left[\mathscr{R}_{x_{0}}^{j-k-1} \frac{\partial \mathscr{R}}{\partial \bar{z}_{i}}(0) \mathscr{R}_{x_{0}}^{k}\right] \bar{z}_{i}^{\prime} \mathscr{P} I_{0}\right)\left(Z, Z^{\prime}\right) \\
&\left.\left.\quad+\frac{1}{(4 \pi)^{j}} \frac{b_{i}}{2^{k} k ! \prod_{k+1}^{j}(2 \ell+1)}+\frac{1}{4 \pi(2 k+2)} \bar{z}_{i}^{\prime}\right) \mathscr{P} I_{0}\right)\left(Z, Z^{\prime}\right)
\end{aligned}
$$

For the last two lines, we used that if $s \in N$, then $\mathscr{L}\left(b_{i} s\right)=4 \pi b_{i} s$ (see Theorem 1.10). Thus, by (0-15) and (3-21)-(3-24),

$$
\begin{aligned}
(3-25) A_{k}(0, Z) & =\frac{1}{(4 \pi)^{k}} \frac{1}{2^{k} k !}\left(I_{2 j}\left(\mathscr{L}_{0}^{-1} \mathcal{O}_{2}^{+2}\right)^{j-k-1} \mathscr{L}_{0}-1\left[\bar{z}_{i} \frac{\partial \mathscr{R}}{\partial \bar{z}_{i}}(0)\right] \Re_{x_{0}}^{k} \mathscr{P} I_{0}\right)(0, Z) \\
& =\left(\mathrm{C}_{j}(j)-\mathrm{C}_{j}(k)\right) I_{2 j}\left[\mathscr{R}_{x_{0}}^{j-k-1} \frac{\partial \mathscr{R} .}{\partial \bar{z}_{i}}(0) \mathscr{R}_{x_{0}}^{k}\right] \bar{z}_{i} \mathscr{P}(0, Z) I_{0} .
\end{aligned}
$$


We know that $\left(\bar{z}_{i} \mathscr{P}\right)^{*}=z_{i} \mathscr{P}$ and $\int_{\mathbb{C}^{n}} z_{m} \bar{z}_{q} e^{-\pi|z|^{2}} d Z=\frac{1}{\pi} \delta_{m q}$, so

$$
\begin{aligned}
\left(A_{k_{1}} A_{k_{2}}{ }^{*}\right)(0,0)=\frac{1}{\pi} I_{2 j}[ & \left.\left(\mathrm{C}_{j}(j)-\mathrm{C}_{j}\left(k_{1}\right)\right) \mathscr{R}_{x_{0}}^{j-k_{1}-1} \frac{\partial \mathscr{R}}{\partial \bar{z}_{i}}(0) \mathscr{R}_{x_{0}}^{k_{1}}\right] \\
\times & {\left[\left(\mathrm{C}_{j}(j)-\mathrm{C}_{j}\left(k_{2}\right)\right) \mathscr{R}_{x_{0}}^{j-k_{2}-1} \frac{\partial \mathscr{R} .}{\partial \bar{z}_{i}}(0) \mathscr{R}_{x_{0}}^{k_{2}}\right]^{*} I_{2 j} . }
\end{aligned}
$$

Finally,

$$
\begin{aligned}
\mathrm{I}_{b}(0,0)=\frac{1}{\pi} \mathrm{I}_{2 j}\left[\sum_{k=0}^{j-1}\left(\mathrm{C}_{j}(j)-\mathrm{C}_{j}(k)\right) \Re_{x_{0}}^{j-k-1} \frac{\partial \mathscr{R}}{\partial \bar{z}_{i}}(0) \mathscr{R}_{x_{0}}^{k}\right] \\
\times\left[\sum_{k=0}^{j-1}\left(\mathrm{C}_{j}(j)-\mathrm{C}_{j}(k)\right) \Re_{x_{0}}^{j-k-1} \frac{\partial \mathscr{R} .}{\partial \bar{z}_{i}}(0) \mathscr{R}_{x_{0}}^{k}\right]^{*} I_{2 j}
\end{aligned}
$$

Computation of $\mathrm{I}_{a}(0,0)$ and $\mathrm{I}_{c}(0,0)$. First recall that $\mathrm{I}_{c}(0,0)=\left(\mathrm{I}_{a}(0,0)\right)^{*}$, so we just need to compute $\mathrm{I}_{a}(0,0)$. By the definition of $\mathrm{I}_{a}(0,0)$, for it to be nonzero, it is necessary to have $j \geq 2$, which will be assumed in this paragraph. Let

$$
\begin{aligned}
A_{k, \ell}:= \\
I_{2 j}\left(\mathscr{L}_{0}{ }^{-1} \mathcal{O}_{2}^{+2}\right)^{j-k-\ell-2}\left(\mathscr{L}_{0}{ }^{-1} \mathcal{O}_{3}^{+2}\right)\left(\mathscr{L}_{0}{ }^{-1} \mathcal{O}_{2}^{+2}\right)^{k}\left(\mathscr{L}_{0}{ }^{-1} \mathcal{O}_{3}^{+2}\right)\left(\mathscr{L}_{0}{ }^{-1} \mathcal{O}_{2}^{+2}\right)^{\ell} \mathscr{P} I_{0},
\end{aligned}
$$

the sum $\mathrm{I}_{a}(0,0)$ is then given by

(3-29) $\mathrm{I}_{a}(0,0)=\int_{\mathbb{R}^{2 n}}\left(\sum_{k, \ell} A_{k, \ell}(0, Z)\right)\left(\frac{1}{(4 \pi)^{j}} \frac{1}{2^{j} j !} I_{2 j} \mathscr{R}_{x_{0}}^{j} \mathscr{P} I_{0}\right)^{*}(Z, 0) d v_{T X}(Z)$.

In the following, we will set

$$
\tilde{b}_{i}:=\frac{b_{i}}{2 \pi}
$$

Using the same method as in (1-44) and (3-22)-(3-24), we find that there exist constants $C_{k, \ell}^{1}, C_{k, \ell}^{2}$ given by

$$
\begin{aligned}
C_{k, \ell}^{1} & =\frac{1}{(4 \pi)^{k+\ell+1}} \frac{1}{2^{k+\ell+1}(k+\ell+1) !}, \\
C_{k, \ell}^{2} & =\frac{1}{(4 \pi)^{k+\ell+1}} \frac{1}{2^{\ell} \ell ! \prod_{\ell+1}^{k+\ell+1}(2 s+1)},
\end{aligned}
$$

such that 
(3-32)

$$
\left(\mathscr{L}_{0}^{-1} \mathcal{O}_{3}^{+2}\right)\left(\mathscr{L}_{0}^{-1} \mathcal{O}_{2}^{+2}\right)^{k}\left(\mathscr{L}_{0}^{-1} \mathcal{O}_{3}^{+2}\right)\left(\mathscr{L}_{0}^{-1} \mathcal{O}_{2}^{+2}\right)^{\ell} \mathscr{P} I_{0}
$$

$=\mathscr{L}_{0}^{-1}\left\{\frac{\partial \mathscr{R} .}{\partial z_{i}}(0) \mathscr{R}_{x_{0}}^{k} \frac{\partial \mathscr{R} .}{\partial z_{i^{\prime}}}(0) \mathscr{R}_{x_{0}}^{\ell} C_{k, \ell}^{1} z_{i} z_{i^{\prime}}+\frac{\partial \mathscr{R}}{\partial \bar{z}_{i}}(0) \mathscr{R}_{x_{0}}^{k} \frac{\partial \mathscr{R}}{\partial z_{i^{\prime}}}(0) \mathscr{R}_{x_{0}}^{\ell} C_{k, \ell}^{1} z_{i^{\prime}}\left(\tilde{b}_{i}+\bar{z}_{i}^{\prime}\right)\right.$

$$
\begin{aligned}
& +\frac{\partial \mathscr{R}}{\partial z_{i}}(0) \mathscr{R}_{x_{0}}^{k} \frac{\partial \mathscr{R}}{\partial \bar{z}_{i^{\prime}}}(0) \mathscr{R}_{x_{0}}^{\ell} z_{i}\left(C_{k, \ell}^{2} \tilde{b}_{i^{\prime}}+C_{k, \ell}^{1} \bar{z}_{i^{\prime}}^{\prime}\right) \\
& \left.+\frac{\partial \mathscr{R}}{\partial \bar{z}_{i}}(0) \mathscr{R}_{x_{0}}^{k} \frac{\partial \mathscr{R}}{\partial \bar{z}_{i^{\prime}}}(0) \mathscr{R}_{x_{0}}^{\ell}\left(\tilde{b}_{i}+\bar{z}_{i}^{\prime}\right)\left(C_{k, \ell}^{2} \tilde{b}_{i^{\prime}}+C_{k, \ell}^{1} \bar{z}_{i^{\prime}}^{\prime}\right)\right\} \mathscr{P} I_{0}
\end{aligned}
$$

$=\mathscr{L}_{0}^{-1}\left\{\frac{\partial \mathscr{R}}{\partial z_{i}}(0) \mathscr{R}_{x_{0}}^{k} \frac{\partial \mathscr{R} .}{\partial z_{i^{\prime}}}(0) \mathscr{R}_{x_{0}}^{\ell} C_{k, \ell}^{1} z_{i} z_{i^{\prime}}\right.$

$$
\begin{aligned}
& +\frac{\partial \mathscr{R}}{\partial \bar{z}_{i}}(0) \mathscr{R}_{x_{0}}^{k} \frac{\partial \mathscr{R}}{\partial z_{i^{\prime}}}(0) \mathscr{R}_{x_{0}}^{\ell} C_{k, \ell}^{1}\left(\tilde{b}_{i} z_{i^{\prime}}+\frac{\delta_{i i^{\prime}}}{\pi}+z_{i^{\prime}} \bar{z}_{i}^{\prime}\right) \\
& +\frac{\partial \mathscr{R}}{\partial z_{i}}(0) \mathscr{R}_{x_{0}}^{k} \frac{\partial \mathscr{R}}{\partial \bar{z}_{i^{\prime}}}(0) \mathscr{R}_{x_{0}}^{\ell}\left(C_{k, \ell}^{2}\left(\tilde{b}_{i^{\prime}} z_{i}+\frac{\delta_{i i^{\prime}}}{\pi}\right)+C_{k, \ell}^{1} z_{i} \bar{z}_{i^{\prime}}^{\prime}\right) \\
& \left.+\frac{\partial \mathscr{R}}{\partial \bar{z}_{i}}(0) \mathscr{R}_{x_{0}}^{k} \frac{\partial \mathscr{R}}{\partial \bar{z}_{i^{\prime}}}(0) \mathscr{R}_{x_{0}}^{\ell}\left(C_{k, \ell}^{2}\left(\tilde{b}_{i} \tilde{b}_{i^{\prime}}+\bar{z}_{i}^{\prime} \tilde{b}_{i^{\prime}}\right)+C_{k, \ell}^{1}\left(\tilde{b}_{i} \bar{z}_{i^{\prime}}^{\prime}+\bar{z}_{i}^{\prime} \bar{z}_{i^{\prime}}^{\prime}\right)\right)\right\} \mathscr{P} I_{0},
\end{aligned}
$$

Using Theorem 1.10, (1-44) and (3-23), we see that there exist constants $C_{j, k, \ell}^{i}$, $i=3, \ldots, 10$, such that

$$
\begin{aligned}
C_{j, k, \ell}^{3} & =C_{k, \ell}^{1} \frac{1}{(4 \pi)^{j-(k+\ell+1)}} \frac{1}{\prod_{k+\ell+2}^{j}(2 s)}, \\
C_{j, k, \ell}^{4} & =C_{k, \ell}^{1} \frac{1}{(4 \pi)^{j-(k+\ell+1)}} \frac{1}{\prod_{k+\ell+2}^{j}(2 s+1)}, \\
C_{j, k, \ell}^{5} & =C_{k, \ell}^{2} \frac{1}{(4 \pi)^{j-(k+\ell+1)}} \frac{1}{\prod_{k+\ell+2}^{j}(2 s+1)}, \\
C_{j, k, \ell}^{6} & =C_{k, \ell}^{2} \frac{1}{(4 \pi)^{j-(k+\ell+1)}} \frac{1}{\prod_{k+\ell+2}^{j}(2 s)},
\end{aligned}
$$

and

(3-34) $\quad A_{k, \ell}(0, Z)$

$$
\begin{aligned}
=I_{2 j} & \left(\mathscr { R } _ { x _ { 0 } } ^ { j - k - \ell - 2 } \left\{\frac{\partial \mathscr{R} .}{\partial \bar{z}_{i}}(0) \mathscr{R}_{x_{0}}^{k} \frac{\partial \mathscr{R} .}{\partial z_{i^{\prime}}}(0)\left(C_{j, k, \ell}^{3} \frac{\delta_{i i^{\prime}}}{\pi}+C_{j, k, \ell}^{4} \tilde{b}_{i} z_{i^{\prime}}\right)\right.\right. \\
& +\frac{\partial \mathscr{R} .}{\partial z_{i}}(0) \mathscr{R}_{x_{0}}^{k} \frac{\partial \mathscr{R} .}{\partial \bar{z}_{i^{\prime}}}(0)\left(C_{j, k, \ell}^{5} \tilde{b}_{i^{\prime}} z_{i}+\frac{\delta_{i i^{\prime}}}{\pi} C_{j, k, \ell}^{6}\right)+\frac{\partial \mathscr{R} .}{\partial \bar{z}_{i}}(0) \mathscr{R}_{x_{0}}^{k} \frac{\partial \mathscr{R} .}{\partial \bar{z}_{i^{\prime}}}(0) \\
& \left.\left.\times\left(C_{j, k, \ell}^{7} \tilde{b}_{i} \tilde{b}_{i^{\prime}}+C_{j, k, \ell}^{8} \bar{z}_{i} \tilde{b}_{i^{\prime}}+C_{j, k, \ell}^{9} \tilde{b}_{i} \bar{z}_{i^{\prime}}+C_{j, k, \ell}^{10} \bar{z}_{i} \bar{z}_{i^{\prime}}\right)\right\} \mathscr{R}_{x_{0}}^{\ell} \mathscr{P} I_{0}\right)(0, Z)
\end{aligned}
$$




$$
\begin{aligned}
= & I_{2 j} \mathscr{R}_{x_{0}}^{j-k-\ell-2}\left\{\frac{\partial \mathscr{R} .}{\partial \bar{z}_{i}}(0) \mathscr{R}_{x_{0}}^{k} \frac{\partial \mathscr{R} .}{\partial z_{i}}(0) \frac{C_{j, k, \ell}^{3}-C_{j, k, \ell}^{4}}{\pi}\right. \\
& +\frac{\partial \mathscr{R} .}{\partial z_{i}}(0) \mathscr{R}_{x_{0}}^{k} \frac{\partial \mathscr{R}}{\partial \bar{z}_{i}}(0) \frac{C_{j, k, \ell}^{6}-C_{j, k, \ell}^{5}}{\pi} \\
& \left.+\frac{\partial \mathscr{R} .}{\partial \bar{z}_{i}}(0) \mathscr{R}_{x_{0}}^{k} \frac{\partial \mathscr{R}}{\partial \bar{z}_{i^{\prime}}}(0)\left(4 C_{j, k, \ell}^{7}-2 C_{j, k, \ell}^{8}-2 C_{j, k, \ell}^{9}+C_{j, k, \ell}^{10}\right) \bar{z}_{i} \bar{z}_{i^{\prime}}\right\} \mathscr{R}_{x_{0}}^{\ell} \mathscr{P}(0, Z) I_{0} .
\end{aligned}
$$

Now with $\int \bar{z}_{i} \bar{z}_{i^{\prime}} \mathscr{P}(0, Z) \mathscr{P}(Z, 0) d Z=0$, we can rewrite (3-29),

$$
\begin{aligned}
\mathrm{I}_{a}(0,0)=\frac{\mathrm{C}_{j}(j)}{\pi} I_{2 j} & \sum_{k, \ell} \mathscr{R}_{x_{0}}^{j-k-\ell-2}\left\{\left(C_{j, k, \ell}^{3}-C_{j, k, \ell}^{4}\right) \frac{\partial \mathscr{R}}{\partial \bar{z}_{i}}(0) \mathscr{R}_{x_{0}}^{k} \frac{\partial \mathscr{R} .}{\partial z_{i}}(0)\right. \\
& \left.+\left(C_{j, k, \ell}^{6}-C_{j, k, \ell}^{5}\right) \frac{\partial \mathscr{R} .}{\partial z_{i}}(0) \mathscr{R}_{x_{0}}^{k} \frac{\partial \mathscr{R} .}{\partial \bar{z}_{i}}(0)\right\} \mathscr{R}_{x_{0}}^{\ell}\left(\mathscr{R}_{x_{0}}^{j}\right)^{*} I_{2 j} .
\end{aligned}
$$

By (0-15), (3-31) and (3-33),

$$
\begin{aligned}
& C_{j, k, \ell}^{3}=\mathrm{C}_{j}(j), \quad C_{j, k, \ell}^{4}=\mathrm{C}_{j}(k+\ell+1), \\
& C_{j, k, \ell}^{5}=\mathrm{C}_{j}(\ell), \quad C_{j, k, \ell}^{6}=\mathrm{C}_{j}(\ell) \prod_{s=k+\ell+2}^{j}\left(1+\frac{1}{2 s}\right) .
\end{aligned}
$$

We can now write $\mathrm{I}_{a}(0,0)$ in (3-35) more precisely as

$$
\begin{aligned}
& \frac{\mathrm{C}_{j}(j)}{\pi} I_{2 j} \sum_{q=0}^{j-2} \sum_{m=0}^{q}\left\{\left(\mathrm{C}_{j}(j)-\mathrm{C}_{j}(q+1)\right) \mathscr{R}_{x_{0}}^{j-(q+2)} \frac{\partial \mathscr{R} .}{\partial \bar{z}_{i}}(0) \Re_{x_{0}}^{q-m} \frac{\partial \mathscr{R} .}{\partial z_{i}}(0) \mathscr{R}_{x_{0}}^{m}\right. \\
& \left.+\mathrm{C}_{j}(m)\left[\prod_{q+2}^{j}\left(1+\frac{1}{2 s}\right)-1\right] \mathscr{R}_{x_{0}}^{j-(q+2)} \frac{\partial \mathscr{R} .}{\partial z_{i}}(0) \mathscr{R}_{x_{0}}^{q-m} \frac{\partial \mathscr{R} .}{\partial \bar{z}_{i}}(0) \mathscr{R}_{x_{0}}^{m}\right\}\left(\mathscr{R}_{x_{0}}^{j}\right)^{*} I_{2 j} .
\end{aligned}
$$

Computation of $\mathrm{II}(0,0)$. Recall that $\mathrm{II}(0,0)=\mathrm{II}_{a}(0,0)+\left(\mathrm{II}_{a}(0,0)\right)^{*}$. The computation of $\mathrm{II}_{a}(0,0)$ is very similar to that of $\mathrm{I}_{a}(0,0)$, only simpler. We will follow the same method.

Let

$$
B_{k}:=I_{2 j}\left(\mathscr{L}_{0}^{-1} \mathcal{O}_{2}^{+2}\right)^{j-k-1}\left(\mathscr{L}_{0}^{-1} \mathcal{O}_{4}^{+2}\right)\left(\mathscr{L}_{0}^{-1} \mathcal{O}_{2}^{+2}\right)^{k} \mathscr{P} I_{0}
$$

the sum $\operatorname{II}_{a}(0,0)$ is then given by

(3-38) $\mathrm{II}_{a}(0,0)=\int_{\mathbb{R}^{2 n}}\left(\sum_{k} B_{k}(0, Z)\right)\left(\frac{1}{(4 \pi)^{j}} \frac{1}{2^{j} j !} I_{2 j} \Re_{x_{0}}^{j} \mathscr{P} I_{0}\right)^{*}(Z, 0) d v_{T X}(Z)$. 
Using (3-19), we can repeat what we have done for (3-32) and (3-34). We find that there is a constant $C$ (which we do not need to compute) such that

$$
\begin{aligned}
B_{k}(0, Z)=I_{2 j}\left\{\mathscr{R}_{x_{0}}^{j-(k+1)} \frac{\partial^{2} \mathscr{R} .}{\partial z_{i} \partial \bar{z}_{i}}(0) \mathscr{R}_{x_{0}}^{k} \frac{\mathrm{C}_{j}(j)-\mathrm{C}_{j}(k)}{\pi}\right. & \\
& \left.+\mathscr{R}_{x_{0}}^{j-(k+1)} \frac{\partial^{2} \mathscr{R} .}{\partial \bar{z}_{i} \partial \bar{z}_{i^{\prime}}}(0) \mathscr{R}_{x_{0}}^{k} C \frac{\bar{z}_{i} \bar{z}_{i^{\prime}}}{2}\right\} \mathscr{P}(0, Z) I_{0} .
\end{aligned}
$$

Thus, we get

$$
\mathrm{II}_{a}(0,0)=\frac{\mathrm{C}_{j}(j)}{\pi} I_{2 j} \sum_{k=0}^{j-1}\left(\mathrm{C}_{j}(j)-\mathrm{C}_{j}(k)\right) \mathscr{R}_{x_{0}}^{j-(k+1)} \frac{\partial^{2} \mathscr{R} .}{\partial z_{i} \partial \bar{z}_{i}}(0) \Re_{x_{0}}^{k}\left(\Re_{x_{0}}^{j}\right)^{*} I_{2 j} .
$$

Conclusion. In order to conclude the proof of Theorem 0.7, we just need to put the pieces together. But before that, to write the formulas in a more intrinsic way, note that we trivialized $\Lambda^{0, \bullet}\left(T^{*} X\right) \otimes E$ with $\nabla^{\Lambda^{0, \bullet} \otimes E}$ and $\bar{w}_{i}=\sqrt{2} \frac{\partial}{\partial \bar{z}_{i}}$, so [Ma and Marinescu 2012, Equations (5.44), (5.45)] imply

$$
\begin{gathered}
\frac{\partial \mathscr{R} .}{\partial \bar{z}_{i}}(0)=\frac{1}{\sqrt{2}}\left(\nabla_{\bar{w}_{i}}^{\Lambda^{0, \bullet} \otimes E_{\mathscr{R}}}\right)\left(x_{0}\right), \quad \frac{\partial \mathscr{R} .}{\partial z_{i}}(0)=\frac{1}{\sqrt{2}}\left(\nabla_{w_{i}}^{\Lambda^{0, \bullet} \otimes E_{\mathscr{R}}}\right)\left(x_{0}\right), \\
\frac{\partial^{2} \mathscr{R} .}{\partial z_{i} \partial \bar{z}_{i}}(0)=-\frac{1}{4}\left(\Delta^{\Lambda^{0, \bullet} \otimes E_{\mathscr{R}}}\right)\left(x_{0}\right) .
\end{gathered}
$$

With these remarks and equations (3-3), (3-17), (3-27), (3-37), (3-40) used in decomposition (3-2), we get Theorem 0.7 .

\section{The third coefficient in the asymptotic expansion when the first two vanish}

In this section, we prove Theorem 0.8. Using (2-6), we know that

$$
I_{2 j} \boldsymbol{b}_{2 j+2} I_{2 j}(0,0)=I_{2 j} \mathscr{F}_{4 j+4} I_{2 j}(0,0) .
$$

Here again, we will first decompose this into several terms in Section 4A, and then in Sections 4B, 4C and 4D we handle them separately.

Fix $j \in \llbracket 1, n \rrbracket$ and suppose that

$$
I_{2 j} \boldsymbol{b}_{2 j} I_{2 j}(0,0)=I_{2 j} \boldsymbol{b}_{2 j+1} I_{2 j}(0,0)=0 .
$$

By Theorems 0.4 and 0.7, this is equivalent to

$$
\left\{\begin{array}{l}
\mathscr{R}_{x}^{j}=0 \\
\mathcal{T}_{0}(j)=0 .
\end{array}\right.
$$

For every smoothing operator $F$ acting on $L^{2}\left(\mathbb{R}^{2 n}, \mathbb{E}_{x_{0}}\right)$ that appears in this section, we will denote by $F\left(Z, Z^{\prime}\right)$ its smooth kernel with respect to $d v_{T X}\left(Z^{\prime}\right)$. 
Moreover, recall that for every operator $A$ we have

$$
\operatorname{Pos}[A]=A A^{*} \quad \text { and } \quad \operatorname{Sym}[A]=A+A^{*} .
$$

4A. Decomposition of the computation. With the same reasoning as in Section 3A, we see that the nonzero terms $\int_{\delta} T_{r}^{\eta}(\lambda) d \lambda$ satisfy $k=2 j, 2 j+1$ or $2 j+2$ in the decomposition (2-11) of $I_{2 j} \mathscr{F}_{4 j+4} I_{2 j}$. Moreover, we can find the possible terms by adding one term to or modifying the subscript of the terms we mentioned in Section 3A. The list of possible terms is as follows:

(I) Terms satisfying $k=2 j+2$.

Here there are up to three indices $i \in\{j, j+1, j+2\}$ such that $\eta_{i}=N$. Moreover, the only $\mathcal{O}_{\ell}$ appearing are some $\mathcal{O}_{2}$. The possibilities are now

(I-a) $2 j+2$ times $\mathcal{O}_{2}^{ \pm 2}$,

(I-a) $2 j$ times $\mathcal{O}_{2}^{ \pm 2}$ and 2 times $\mathcal{O}_{2}^{0}$.

(II) Terms satisfying $k=2 j+1$.

Here, there are one or two indices $i \in\{j, j+1\}$ such that $\eta_{i}=N$, and there is exactly one $\mathcal{O}_{\ell}^{0}$ that appears in these terms. We regroup them in relation to the $\mathcal{O}_{r_{i}}$ that they contain:

(II-b) $2 j$ times $\mathcal{O}_{2}^{ \pm 2}$ and 1 time $\mathcal{O}_{4}^{0}$,

(II-b) $2 j-1$ times $\mathcal{O}_{2}^{ \pm 2}, 1$ time $\mathcal{O}_{2}^{0}$ and 1 time $\mathcal{O}_{4}^{ \pm 2}$,

(II-b) $2 j-1$ times $\mathcal{O}_{2}^{ \pm 2}, 1$ time $\mathcal{O}_{3}^{ \pm 2}$ and 1 time $\mathcal{O}_{3}^{0}$,

(II-b) $2 j-2$ times $\mathcal{O}_{2}^{ \pm 2}, 1$ time $\mathcal{O}_{2}^{0}$ and 2 times $\mathcal{O}_{3}^{ \pm 2}$.

(III) Terms satisfying $k=2 j$.

Here, the $i_{0}$ such that $\eta_{i_{0}}=N$ is unique and equal to $j$, and no $\mathcal{O}_{\ell}^{0}$ appears in these terms. We regroup them in relation to the $\mathcal{O}_{r_{i}}$ that they contain:

(III-c) $2 j-4$ times $\mathcal{O}_{2}^{ \pm 2}$ and 4 times $\mathcal{O}_{3}^{ \pm 2}$,

(III-c) $2 j-3$ times $\mathcal{O}_{2}^{ \pm 2}, 2$ times $\mathcal{O}_{3}^{ \pm 2}$ and 1 time $\mathcal{O}_{4}^{ \pm 2}$,

(III-c) $2 j-2$ times $\mathcal{O}_{2}^{ \pm 2}$ and 2 times $\mathcal{O}_{4}^{ \pm 2}$,

(III-c) $2 j-2$ times $\mathcal{O}_{2}^{ \pm 2}, 1$ time $\mathcal{O}_{3}^{ \pm 2}$ and 1 time $\mathcal{O}_{5}^{ \pm 2}$,

(III-c) $2 j-1$ times $\mathcal{O}_{2}^{ \pm 2}$ and 1 time $\mathcal{O}_{6}^{ \pm 2}$.

This list seems quite long, but fortunately most of the terms will ultimately vanish due to the fact that they are computed by means of some terms involved in $I_{2 j} \boldsymbol{b}_{2 j} I_{2 j}$ and $I_{2 j} \boldsymbol{b}_{2 j+1} I_{2 j}$.

In the sequel, the contribution to the third coefficient of the terms of type I-a), I-b), etc., will be denoted by $T_{\mathrm{I}-\mathrm{a})}, T_{\mathrm{I}-\mathrm{b})}$, etc.

4B. Terms of type I. We begin with an observation, whose proof is an easy extension of the computation (2-21), using the fact that $\mathscr{R}_{x}^{j}=0$. 
Lemma 4.1. For any $j$-tuple $\left(a_{1}, \ldots, a_{j}\right)$ of positive integers, we have

$$
X_{\left(a_{1}, \ldots, a_{j}\right)}:=I_{2 j}\left(\prod_{i=1}^{j} \mathscr{L}_{0}{ }^{-a_{i}} \mathcal{O}_{2}^{+2}\right) P^{N}=0 .
$$

Terms of type I-a). In these terms, only some $\mathcal{O}_{2}^{ \pm 2}$ appear. So there is either a unique $i_{0}$ such that $\eta_{i_{0}}=N$ which is then equal to $j$ or $j+2$, or exactly two such $i_{0}$ which are then $j$ and $j+2$.

Each term in the second case is a sum of terms of the form

$$
-X_{\left(a_{1}, \ldots, a_{j}\right)} \mathcal{O}_{2}^{-2} \mathscr{L}_{0}^{-b} \mathcal{O}_{2}^{+2} X_{\left(a_{1}^{\prime}, \ldots, a_{j}^{\prime}\right)}^{*}
$$

with $a_{i}, a_{k}^{\prime}, b \in\{1,2\}$ (exactly one is equal to 2 ). By Lemma 4.1, these terms vanish.

Now, each term in the first case is equal or adjoint to a term of the form

$$
I_{2 j}\left(\prod_{i=1}^{j+2} \mathscr{L}_{0}^{-1} \mathcal{O}_{2}^{\varepsilon_{i}}\right) \mathscr{P} I_{0}\left(I_{2 j}\left(\mathscr{L}_{0}^{-1} \mathcal{O}_{2}^{+2}\right)^{j} \mathscr{P} I_{0}\right)^{*}
$$

where $\varepsilon_{i} \in\{-2,+2\}$ (exactly one of the $\varepsilon_{i}$ is equal to -2 ). By Lemma 4.1, these terms vanish.

Finally, every term of type I-a) vanishes and $T_{\mathrm{I}-\mathrm{a})}=0$.

Terms of type I-b). Using Lemma 4.1 as above, we see that the only nonzero terms of this type satisfy the condition that before the first index $i$ such that $\eta_{i}=N$ and after the last, there must be a $\mathcal{O}_{2}^{0}$ appearing. As a consequence, the cases where two or three $\eta_{i}$ are equal to $N$ lead to vanishing terms. We now deal with the terms where $\eta_{j+1}=N$ and for $i \neq j+1, \eta_{i}=N^{\perp}$. Such terms are of the form

$$
\begin{aligned}
\left(I_{2 j}\left(\mathscr{L}_{0}{ }^{-1} \mathcal{O}_{2}^{+2}\right)^{j-k}\right. & \left.\mathscr{L}_{0}{ }^{-1} \mathcal{O}_{2}^{0}\left(\mathscr{L}_{0}^{-1} \mathcal{O}_{2}^{+2}\right)^{k} \mathscr{P}\right) \\
& \times\left(I_{2 j}\left(\mathscr{L}_{0}^{-1} \mathcal{O}_{2}^{+2}\right)^{j-k^{\prime}} \mathscr{L}_{0}{ }^{-1} \mathcal{O}_{2}^{0}\left(\mathscr{L}_{0}{ }^{-1} \mathcal{O}_{2}^{+2}\right)^{k^{\prime}} \mathscr{P}\right)^{*}
\end{aligned}
$$

for $0 \leq k, k^{\prime} \leq j$. By the computations in Section 3B, an in particular (3-16), we find

$$
\begin{aligned}
& I_{2 j}\left(\left(\mathscr{L}_{0}^{-1} \mathcal{O}_{2}^{+2}\right)^{j-k} \mathscr{L}_{0}{ }^{-1} \mathcal{O}_{2}^{0}\left(\mathscr{L}_{0}^{-1} \mathcal{O}_{2}^{+2}\right)^{k}\right) \mathscr{P} I_{0} \\
= & I_{2 j} \mathscr{R}_{x}^{j-k}\left[\frac{1}{6}\left(\mathrm{C}_{j+1}(j+1)-\frac{\mathrm{C}_{j}(k)}{2 \pi(2 k+1)}\right) r_{x}^{X}-\frac{\mathrm{C}_{j}(k)}{4 \pi(2 k+1)} \sqrt{-1} R_{\Lambda, x}^{E}\right] \mathscr{R}_{x}^{k} \mathscr{P} I_{0} .
\end{aligned}
$$

Observe that $r^{X}$ commutes with $\mathscr{R}$ and $\mathscr{R}^{j}=0$. So the contribution of the terms of type I-b) is finally $T_{\mathrm{I}-\mathrm{b})}=\operatorname{Pos}\left[\mathcal{T}_{3}^{\prime}(j)\right]$.

\section{C. Terms of type II.}

Terms of type II-a). In these terms, there are either only $\mathcal{O}_{2}^{-2}$ appearing at the right of the first $P^{N}$ or only $\mathcal{O}_{2}^{+2}$ appearing at the left of the last $P^{N}$. Either way, all these terms vanish by Lemma 4.1. Hence $T_{\mathrm{II}-\mathrm{a})}=0$. 
Terms of type II-b). For these terms, there are two possibilities.

Firstly, there are two indices $i$ such that $\eta_{i}=N$, and then they are equal to $j$ and $j+1$. In this case, either before the first $P^{N}$ or after the last, there appear $j \mathcal{O}_{2}^{+2}$ (or $\mathcal{O}_{2}^{-2}$ ), so all these terms vanish.

Secondly, there is a unique $i_{0}$ such that $\eta_{i_{0}}=N$ and it is equal to $j$ or $j+1$. We denote by $S_{1}$ (resp. $S_{2}$ ) the sum of the terms for which $i_{0}=j\left(\right.$ resp. $\left.i_{0}=j+1\right)$. Then $S_{1}=S_{2}^{*}$ and

$$
\begin{aligned}
S_{2}=\sum_{k, \ell}\left\{I_{2 j}\left(\mathscr{L}_{0}^{-1} \mathcal{O}_{2}^{+2}\right)^{j-k-1}\left(\mathscr{L}_{0}^{-1} \mathcal{O}_{4}^{+2}\right)\left(\mathscr{L}_{0}^{-1} \mathcal{O}_{2}^{+2}\right)^{k} \mathscr{P} I_{0}\right\} \\
\times\left\{I_{2 j}\left(\mathscr{L}_{0}^{-1} \mathcal{O}_{2}^{+2}\right)^{j-\ell} \mathscr{L}_{0}{ }^{-1} \mathcal{O}_{2}^{0}\left(\mathscr{L}_{0}{ }^{-1} \mathcal{O}_{2}^{+2}\right)^{\ell} \mathscr{P} I_{0}\right\}^{*} \\
=\left\{\sum_{k} I_{2 j}\left(\mathscr{L}_{0}{ }^{-1} \mathcal{O}_{2}^{+2}\right)^{j-k-1}\left(\mathscr{L}_{0}^{-1} \mathcal{O}_{4}^{+2}\right)\left(\mathscr{L}_{0}^{-1} \mathcal{O}_{2}^{+2}\right)^{k} \mathscr{P} I_{0}\right\} \\
\times\left\{\sum_{\ell} I_{2 j}\left(\mathscr{L}_{0}^{-1} \mathcal{O}_{2}^{+2}\right)^{j-\ell} \mathscr{L}_{0}{ }^{-1} \mathcal{O}_{2}^{0}\left(\mathscr{L}_{0}{ }^{-1} \mathcal{O}_{2}^{+2}\right)^{\ell} \mathscr{P} I_{0}\right\}^{*}
\end{aligned}
$$

By (3-16) and (3-40) we find that the contribution of the terms of type II-b), i.e., $S_{1}(0,0)+S_{2}(0,0)$, is $T_{\text {II-b) }}=\operatorname{Sym}\left[\mathcal{T}_{2}(j) \mathcal{T}_{3}^{\prime}(j)^{*}\right]$.

Terms of type II-c). The computation is the same as for terms of type II-b), except that in the case of a unique $i_{0}$ such that $\eta_{i_{0}}=N$, we must replace $\mathcal{O}_{4}^{+2}$ by $\mathcal{O}_{3}^{+2}$ and $\mathcal{O}_{2}^{0}$ by $\mathcal{O}_{3}^{0}$ in (4-10). Recall that $A_{k}$ has been defined in (3-20). By (3-25) and (4-3), we find that the contribution of the terms of type II-c) is the symmetric operator associated to

$$
\left\{\sum_{k} A_{k}\right\}\left\{\sum_{\ell} I_{2 j}\left(\mathscr{L}_{0}^{-1} \mathcal{O}_{2}^{+2}\right)^{j-\ell} \mathscr{L}_{0}^{-1} \mathcal{O}_{3}^{0}\left(\mathscr{L}_{0}^{-1} \mathcal{O}_{2}^{+2}\right)^{\ell} \mathscr{P} I_{0}\right\}^{*}
$$

By (4-3) we get $T_{\mathrm{II}-\mathrm{c})}=0$.

Terms of type II-d). Here again, we have the same possibilities concerning the indices $i$ such that $\eta_{i}=N$ as for terms of types II-b) or II-c). If there are two such indices, then they are equal to $j$ and $j+1$ and between the two corresponding $P^{N}$ we will have the term $\mathcal{O}_{2}^{0}$. By (3-5), these terms vanish.

We now suppose that there is a unique $i_{0}$ such that $\eta_{i_{0}}=N$. Then $i_{0}=j$ or $j+1$. As $\mathscr{R}_{x}^{j}=0$, any term in which the two $\mathcal{O}_{3}$ and the $\mathcal{O}_{2}^{0}$ appear on the same side of $P^{N}$ will vanish. A term with one $\mathcal{O}_{3}$ at the left and one $\mathcal{O}_{3}$ at the right of $P^{N}$ is equal or adjoint to

$$
I_{2 j}\left(\prod_{i=1}^{j+1} \mathscr{L}_{0}^{-1} \mathcal{O}_{a_{i}}^{\varepsilon_{i}}\right) \mathscr{P} I_{0} \times A_{k}^{*}
$$


where $a_{i}=2$ or 3 and $\varepsilon_{i}=+2$ except for exactly one $i_{1}$ satisfying $a_{i_{1}}=2$ (for which $\left.\varepsilon_{i_{1}}=0\right)$. By (3-25) and (4-3), the sum of these terms vanishes.

Finally, the other possibility is that the two $\mathcal{O}_{3}$ appear on the same side of $P^{N}$, and $\mathcal{O}_{2}^{0}$ on the other side. Recall that $A_{k, \ell}$ has been defined in (3-28). The sum of the remaining terms is equal to

$$
\operatorname{Sym}\left[\left\{\sum_{k, \ell} A_{k, \ell}\right\}\left\{\sum_{m} I_{2 j}\left(\mathscr{L}_{0}^{-1} \mathcal{O}_{2}^{+2}\right)^{j-m} \mathscr{L}_{0}^{-1} \mathcal{O}_{2}^{0}\left(\mathscr{L}_{0}^{-1} \mathcal{O}_{2}^{+2}\right)^{m} \mathscr{P} I_{0}\right\}^{*}\right] .
$$

As a result, the contribution of terms of type II-d) is $T_{\mathrm{II}-\mathrm{d})}=\operatorname{Sym}\left[\mathcal{T}_{1}(j) \mathcal{T}_{3}^{\prime}(j)^{*}\right]$.

4D. Terms of type III. The computations rely on similar arguments as in Sections 4B and 4C. We will therefore give the contribution of each sub-type directly.

Terms of type III-a). The contribution is $T_{\mathrm{III}-\mathrm{a})}=\operatorname{Pos}\left[\mathcal{T}_{1}(j)\right]$.

Terms of type III-b). The contribution is $T_{\text {III-b) }}=\operatorname{Sym}\left[\mathcal{T}_{1}(j) \mathcal{T}_{2}(j)^{*}\right]$

Terms of type III-c). The contribution is $T_{\mathrm{III}-\mathrm{c})}=\operatorname{Pos}\left[\mathcal{T}_{2}(j)\right]$.

Terms of type III-d). The sum of all these terms vanishes, $T_{\text {III-d) }}=0$.

Terms of type III-e). These terms vanish, so that $T_{\text {III-e) }}=0$.

By all the computations in Sections $4 \mathrm{~B}, 4 \mathrm{C}$ and $4 \mathrm{D}$, we get Theorem 0.8 .

\section{Acknowledgments}

The authors want to thank Professor Xiaonan Ma for helpful discussions about this topic, and more generally for his kind and constant support.

\section{References}

[Catlin 1999] D. Catlin, "The Bergman kernel and a theorem of Tian", pp. 1-23 in Analysis and geometry in several complex variables (Katata, 1997), edited by G. Komatsu and M. Kuranishi, Birkhäuser, Boston, 1999. MR 2000e:32001 Zbl 0941.32002

[Charbonneau and Stern 2011] B. Charbonneau and M. Stern, "Asymptotic Hodge theory of vector bundles", preprint, 2011. arXiv 1111.0591v1

[Dai et al. 2006] X. Dai, K. Liu, and X. Ma, "On the asymptotic expansion of Bergman kernel", $J$. Differential Geom. 72:1 (2006), 1-41. MR 2007k:58043 Zbl 1099.32003

[Donaldson 2001] S. K. Donaldson, "Scalar curvature and projective embeddings, I", J. Differential Geom. 59:3 (2001), 479-522. MR 2003j:32030 Zbl 1052.32017

[Fine 2012] J. Fine, "Quantization and the Hessian of Mabuchi energy", Duke Math. J. 161:14 (2012), 2753-2798. MR 2993140 Zbl 1262.32024 arXiv 1009.4543

[Lu 2000] Z. Lu, "On the lower order terms of the asymptotic expansion of Tian-Yau-Zelditch", Amer. J. Math. 122:2 (2000), 235-273. MR 2002d:32034 Zbl 0972.53042

[Ma 2011] X. Ma, "Geometric quantization on Kähler and symplectic manifolds", pp. 785-810 in Proceedings of the International Congress of Mathematicians (Hyderabad, 2010), vol. II, edited by

R. Bhatia et al., Hindustan Book Agency, New Delhi, 2011. MR 2012g:53188 Zbl 1229.53088 
[Ma and Marinescu 2002] X. Ma and G. Marinescu, "The $\operatorname{spin}^{c}$ Dirac operator on high tensor powers of a line bundle", Math. Z. 240:3 (2002), 651-664. MR 2003h:58043 Zbl 1027.58025

[Ma and Marinescu 2006] X. Ma and G. Marinescu, "The first coefficients of the asymptotic expansion of the Bergman kernel of the spin ${ }^{c}$ Dirac operator", Int. J. Math. 17:6 (2006), 737-759. MR 2007c:58040 Zbl 1106.58018

[Ma and Marinescu 2007] X. Ma and G. Marinescu, Holomorphic Morse inequalities and Bergman kernels, Progress in Mathematics 254, Birkhäuser, Basel, 2007. MR 2008g:32030 Zbl 1135.32001

[Ma and Marinescu 2008] X. Ma and G. Marinescu, "Generalized Bergman kernels on symplectic manifolds", Adv. Math. 217:4 (2008), 1756-1815. MR 2009c:58047 Zbl 1141.58018

[Ma and Marinescu 2012] X. Ma and G. Marinescu, "Berezin-Toeplitz quantization on Kähler manifolds", J. Reine Angew. Math. 662 (2012), 1-56. MR 2876259 Zbl 1251.47030

[Tian 1990] G. Tian, "On a set of polarized Kähler metrics on algebraic manifolds", J. Differential Geom. 32:1 (1990), 99-130. MR 91j:32031 Zbl 0706.53036

[Wang 2003] L. Wang, Bergman kernel and stability of holomorphic vector bundles with sections, thesis, Massachusetts Institute of Technology, Cambridge, MA, 2003, Available at http://hdl.handle.net/ 1721.1/29983. MR 2717131

[Wang 2005] X. Wang, "Canonical metrics on stable vector bundles", Comm. Anal. Geom. 13:2 (2005), 253-285. MR 2006b:32031 Zbl 1093.32008

[Xu 2012] H. Xu, "A closed formula for the asymptotic expansion of the Bergman kernel", Comm. Math. Phys. 314:3 (2012), 555-585. MR 2964769 Zbl 1257.32003 arXiv 1103.3060

[Zelditch 1998] S. Zelditch, "Szegő kernels and a theorem of Tian", Int. Math. Res. Notices 1998:6 (1998), 317-331. MR 99g:32055 Zbl 0922.58082

Received January 10, 2014. Revised July 22, 2014.

MARTIN PUCHOL

UFR DE MATHÉMATIQUES

UNIVERSITÉ DIDEROT PARIS 7

CAMPUS DES GRANDS MOULINS

BÂTIMENT SOPHIE GERMAIN

5 Rue Thomas Mann, Case 7012

75205 PARIS CEDEX 13

FRANCE

martin.puchol@imj-prg.fr

JIALIN ZHU

Chern Institute of Mathematics

NANKAI UNIVERSITY

TIANJIN, 300071

CHINA

jialinzhu@nankai.edu.cn 


\title{
PACIFIC JOURNAL OF MATHEMATICS
}

\author{
msp.org/pjm
}

Founded in 1951 by E. F. Beckenbach (1906-1982) and F. Wolf (1904-1989)

\section{EDITORS}

Don Blasius (Managing Editor)

Department of Mathematics

University of California

Los Angeles, CA 90095-1555

blasius@math.ucla.edu

\author{
Paul Balmer \\ Department of Mathematics \\ University of California \\ Los Angeles, CA 90095-1555 \\ balmer@math.ucla.edu \\ Robert Finn \\ Department of Mathematics \\ Stanford University \\ Stanford, CA 94305-2125 \\ finn@math.stanford.edu \\ Sorin Popa \\ Department of Mathematics \\ University of California \\ Los Angeles, CA 90095-1555 \\ popa@math.ucla.edu
}

\author{
Vyjayanthi Chari \\ Department of Mathematics \\ University of California \\ Riverside, CA 92521-0135 \\ chari@math.ucr.edu \\ Kefeng Liu \\ Department of Mathematics \\ University of California \\ Los Angeles, CA 90095-1555 \\ liu@math.ucla.edu \\ Jie Qing \\ Department of Mathematics \\ University of California \\ Santa Cruz, CA 95064 \\ qing@ cats.ucsc.edu
}

\section{PRODUCTION}

Silvio Levy, Scientific Editor, production@msp.org

\section{SUPPORTING INSTITUTIONS}

ACADEMIA SINICA, TAIPEI

CALIFORNIA INST. OF TECHNOLOGY

INST. DE MATEMÁTICA PURA E APLICADA

KEIO UNIVERSITY

MATH. SCIENCES RESEARCH INSTITUTE

NEW MEXICO STATE UNIV.

OREGON STATE UNIV.

\author{
STANFORD UNIVERSITY \\ UNIV. OF BRITISH COLUMBIA \\ UNIV. OF CALIFORNIA, BERKELEY \\ UNIV. OF CALIFORNIA, DAVIS \\ UNIV. OF CALIFORNIA, LOS ANGELES \\ UNIV. OF CALIFORNIA, RIVERSIDE \\ UNIV. OF CALIFORNIA, SAN DIEGO \\ UNIV. OF CALIF., SANTA BARBARA
}

\author{
Daryl Cooper \\ Department of Mathematics \\ University of California \\ Santa Barbara, CA 93106-3080 \\ cooper@math.ucsb.edu \\ Jiang-Hua Lu \\ Department of Mathematics \\ The University of Hong Kong \\ Pokfulam Rd., Hong Kong \\ jhlu@maths.hku.hk \\ Paul Yang \\ Department of Mathematics \\ Princeton University \\ Princeton NJ 08544-1000 \\ yang@math.princeton.edu
}

These supporting institutions contribute to the cost of publication of this Journal, but they are not owners or publishers and have no responsibility for its contents or policies.

See inside back cover or msp.org/pjm for submission instructions.

The subscription price for 2015 is US \$420/year for the electronic version, and \$570/year for print and electronic.

Subscriptions, requests for back issues and changes of subscribers address should be sent to Pacific Journal of Mathematics, P.O. Box 4163, Berkeley, CA 94704-0163, U.S.A. The Pacific Journal of Mathematics is indexed by Mathematical Reviews, Zentralblatt MATH, PASCAL CNRS Index, Referativnyi Zhurnal, Current Mathematical Publications and Web of Knowledge (Science Citation Index).

The Pacific Journal of Mathematics (ISSN 0030-8730) at the University of California, c/o Department of Mathematics, 798 Evans Hall \#3840, Berkeley, CA 94720-3840, is published twelve times a year. Periodical rate postage paid at Berkeley, CA 94704, and additional mailing offices. POSTMASTER: send address changes to Pacific Journal of Mathematics, P.O. Box 4163, Berkeley, CA 94704-0163.

PJM peer review and production are managed by EditFLOW ${ }^{\circledR}$ from Mathematical Sciences Publishers.

\section{PUBLISHED BY}

\section{mathematical sciences publishers \\ nonprofit scientific publishing}

http://msp.org/

(C) 2015 Mathematical Sciences Publishers 


\section{PACIFIC JOURNAL OF MATHEMATICS}

Volume $274 \quad$ No. $2 \quad$ April 2015

On Demazure and local Weyl modules for affine hyperalgebras

Angelo Bianchi, Tiago Macedo and Adriano Moura

On curves and polygons with the equiangular chord property

305

TARik Aougab, Xidian Sun, Serge TabachniKov and

YUWEN WANG

The well-posedness of nonlinear Schrödinger equations in Triebel-type 325 spaces

SHAOLEI RU and JiECHENG CHEN

Hypersurfaces with constant curvature quotients in warped product manifolds

JIE WU and CHAO XIA

The first terms in the expansion of the Bergman kernel in higher degrees

MARTin PUCHOL and JiAlin ZHU

Determinant rank of $C^{*}$-algebras

Guinua Gong, HuAXIN Lin and Yifeng XUE

Motion by mixed volume preserving curvature functions near spheres

437

DAVID HARTLEY

Homomorphisms on infinite direct products of groups, rings and monoids

GEORGE M. BERGMAN

The virtual first Betti number of soluble groups

Martin R. BRIDSON and Dessislava H. KochloukOVA 